\title{
Modeling interactions of photons with pseudoscalar and vector mesons
}

\author{
Henryk Czyż, ${ }^{1}$ Patrycja Kisza, ${ }^{2}$ and Szymon Tracz ${ }^{2}$ \\ ${ }^{1}$ Institute of Physics, University of Silesia, PL-41500 Chorzów, Poland \\ and Helmholtz-Institut, 55128 Mainz, Germany \\ ${ }^{2}$ Institute of Physics, University of Silesia, PL-41500 Chorzów, Poland
}

(Received 7 November 2017; published 12 January 2018; corrected 23 January 2018)

\begin{abstract}
We model the interaction of photons, pseudoscalars, and vector mesons within resonance chiral symmetric theory with SU(3) breaking. The couplings of the model are fitted to the experimental data. Within the developed model we predict the pseudoscalar-exchange light-by-light contributions to the muon anomalous magnetic moment $a_{\mu}^{P}=(82.8 \pm 3.4) \times 10^{-11}$. The error covers also the model dependence within the class of models considered in this paper. The model was implemented into the Monte Carlo event generator EKHARA to simulate reactions $e^{+} e^{-} \rightarrow e^{+} e^{-} P, P=\pi^{0}, \eta, \eta^{\prime}$ and into the Monte Carlo event generator PHOKHARA to simulate reactions $e^{+} e^{-} \rightarrow P \gamma(\gamma)$.
\end{abstract}

DOI: 10.1103/PhysRevD.97.016006

\section{INTRODUCTION}

During the last several years, many very accurate experimental data, which contain information about photonhadron interactions, emerged. At the same time, one can observe a significant contribution from the theory community to improve the quality of the models used to describe the experimental data. Thus, the quest for precision in hadronphoton interactions [1] is well under way. The two main reasons for this effort, besides the pure interest in knowing better the microscopic world, are the discrepancy at the level of almost $4 \sigma$ between the measured [2] and the calculated [3-8] anomalous magnetic moment of the muon $\left(a_{\mu}\right)$ and the accuracy of the electromagnetic running coupling constant calculated at $M_{Z}$ [3], which is a limiting factor in future tests of the Standard Model. In both cases, the hadronic contributions are the source of the uncertainties as electroweak corrections are well under control.

In this paper, we extend the validity of the model developed in [9] to be able not only to model correctly the $\gamma^{*}-\gamma^{*}-P$ form factors in the spacelike region, which are necessary to calculate the pseudoscalar-exchange lightby-light contributions to the $a_{\mu}[7,10]$, but also to describe correctly all the experimental data which can be predicted from the Lagrangians $\mathcal{L}_{\gamma \gamma \mathcal{P}}, \mathcal{L}_{\gamma V}, \mathcal{L}_{V \gamma P}$, and $\mathcal{L}_{V V P}$. A similar research program of a global fit was carried out within the hidden local symmetry effective Lagrangian $[5,11,12]$ with many statistical tests carried out, yet concentrating on the

Published by the American Physical Society under the terms of the Creative Commons Attribution 4.0 International license. Further distribution of this work must maintain attribution to the author(s) and the published article's title, journal citation, and DOI. Funded by SCOAP ${ }^{3}$. modeling of the processes needed for the calculations of the leading order hadronic vacuum polarization contributions to $a_{\mu}$. We plan to extend our analysis to cover also the $e^{+} e^{-} \rightarrow \pi^{+} \pi^{-}, K^{+} K^{-}, K^{0} \bar{K}^{0}$, and $\pi^{+} \pi^{-} \pi^{0}$ in a future publication. This way it will be possible to study the model dependence of the obtained results comparing the hidden local symmetry and resonance chiral Lagrangian approach, which despite similarities are not identical. The $\gamma^{*}-\gamma^{*}-P$ form factors, one of the outcome of this paper, are modeled within various frameworks [9,13-32]: phenomenology oriented, aiming for model independence Padé approximants, chiral effective resonance theory, quark models, and Nambu-Jona-Lasinio model.

The paper is organized in the following way: In Sec. II we describe the modifications of the model developed in [9]. In Sec. III we describe the fits to experimental data. In Sec. IV the asymptotic behavior and the slopes of the pseudoscalar form factors are discussed. In Sec. V we present the evaluation, within the developed model, of the pseudoscalar-exchange light-by-light contributions to the anomalous magnetic moment of the muon. In Sec. VI the implementations to the Monte Carlo event generators PHOKHARA [33,34] and EKHARA $[35,36]$ are presented. We shortly summarize the results in Sec. VII.

\section{THE MODEL}

As said already in the Introduction, one of the aims of this paper was to extend the model used in [9] for the modeling of the $\gamma^{*}-\gamma^{*}-P$ form factors in the spacelike region to be able to cover also the timelike region, adding to the list of modeled entities also other physical observables (see Sec. III). In [9] the SU(3) isospin symmetry was 
assumed for the couplings in the relevant Lagrangians. However, from the experimental data, which are modeled by the form factors in the timelike region, it is evident that this symmetry is broken (see the discussion in the next section). The strategy to model all spacelike and timelike data was to extend the model from [9] in the minimal possible way to describe the whole set of experimental data. In [9] it was checked that the spacelike data can be modeled using only two vector-meson octets. When extending the model to the timelike region as well, one has to use at least three octets. This was adopted within this paper. The $\eta-\eta^{\prime}$ mixing scheme, which was taken in [9] from [37,38], is kept unchanged. However, as there are new data available, we have fitted the mixing parameters to the experimental observables predicted from the Lagrangians described below.

The Wess-Zumino-Witten Lagrangian [39,40], which describes the interaction of pseudoscalar mesons with two photons, can be written down in the terms of the physical fields as

$$
\mathcal{L}_{\gamma \gamma \mathcal{P}}=\frac{-e^{2} N_{c}}{24 \pi^{2} f_{\pi}} \epsilon^{\mu \nu \alpha \beta} \partial_{\mu} B_{\nu} \partial_{\alpha} B_{\beta}\left[\pi^{0}+\eta\left(\frac{5}{3} C_{q}-\frac{\sqrt{2}}{3} C_{s}\right)+\eta^{\prime}\left(\frac{5}{3} C_{q}^{\prime}+\frac{\sqrt{2}}{3} C_{s}^{\prime}\right)\right] .
$$

The $\gamma V$ interaction is described in terms of the following Lagrangian:

$$
\mathcal{L}_{\gamma V}=-e \sum_{i=1}^{3} f_{V_{i}} \partial_{\mu} B_{\nu}\left(\widetilde{\rho}_{i}^{\mu \nu}+\frac{1}{3} F_{\omega_{i}} \tilde{\omega}_{i}^{\mu \nu}-\frac{\sqrt{2}}{3} F_{\phi_{i}} \tilde{\phi}_{i}^{\mu \nu}\right),
$$

where $\tilde{V}_{\mu \nu} \equiv \partial_{\mu} V_{\nu}-\partial_{\nu} V_{\mu}, f_{V_{i}}$ is a dimensionless coupling for the vector representation of the spin-1 fields in a given octet. The SU(3) symmetry of the coupling constants is broken here in the first octet only, by introducing the additional constants $F_{\omega_{1}}$ and $F_{\phi_{1}}$. For the other octets the constants are set to $1: F_{\omega_{i}}=F_{\phi_{i}}=1$, for $i=2$, 3, preserving the SU(3) symmetry in the higher octets.

The Lagrangians that describe the vector-photon-pseudoscalar and two vector mesons interaction with the pseudoscalar come from an extension of the Lagrangians from [41], which was adopted in [9]. In terms of the physical fields they read

$$
\begin{aligned}
& \mathcal{L}_{V \gamma \pi^{0}}=-\sum_{i=1}^{n} \frac{4 \sqrt{2} e h_{V_{i}}}{3 f_{\pi}} \epsilon_{\mu \nu \alpha \beta} \partial^{\alpha} B^{\beta}\left(\rho_{i}^{\mu}+3 H_{\omega_{i}} \omega_{i}^{\mu}-\frac{3}{\sqrt{2}} A_{i}^{\pi_{0}} \phi_{i}^{\mu}\right) \partial^{\nu} \pi^{0}, \\
& \mathcal{L}_{V \gamma \eta}=-\sum_{i=1}^{n} \frac{4 \sqrt{2} e h_{V_{i}}}{3 f_{\pi}} \epsilon_{\mu \nu \alpha \beta} \partial^{\alpha} B^{\beta}\left[\left(3 \rho_{i}^{\mu}+\omega_{i}^{\mu}\right) C_{q}+2 \phi_{i}^{\mu} C_{s}-\left(\frac{5}{\sqrt{2}} C_{q}-C_{s}\right) A_{i}^{\eta} \phi_{i}^{\mu}\right] \partial^{\nu} \eta, \\
& \mathcal{L}_{V \gamma \eta^{\prime}}=-\sum_{i=1}^{n} \frac{4 \sqrt{2} e h_{V_{i}}}{3 f_{\pi}} \epsilon_{\mu \nu \alpha \beta} \partial^{\alpha} B^{\beta}\left[\left(3 \rho_{i}^{\mu}+\omega_{i}^{\mu}\right) C_{q}^{\prime}-2 \phi_{i}^{\mu} C_{s}^{\prime}-\left(\frac{5}{\sqrt{2}} C_{q}^{\prime}+C_{s}^{\prime}\right) A_{i}^{\eta^{\prime}} \phi_{i}^{\mu}\right] \partial^{\nu} \eta^{\prime}, \\
& \mathcal{L}_{V V \pi^{0}}=-\sum_{i=1}^{n} \frac{4 \sigma_{V_{i}}}{f_{\pi}} \epsilon_{\mu \nu \alpha \beta}\left[\frac{1}{F_{\omega_{i}}} \pi^{0} \partial^{\mu} \omega_{i}^{\nu} \partial^{\alpha} \rho_{i}^{\beta}+\frac{3\left(F_{\omega_{i}} H_{\omega_{i}}-1-A_{\phi \omega, i}^{\pi^{0}}\right)}{2 F_{\omega_{i}}^{2}} \pi^{0} \partial^{\mu} \omega_{i}^{\nu} \partial^{\alpha} \omega_{i}^{\beta}\right. \\
& \left.+\frac{3\left(A_{i}^{\pi_{0}}-A_{\phi \omega, i}^{\pi^{0}} / F_{\phi_{i}}\right)}{4 F_{\phi_{i}}} \pi^{0} \partial^{\mu} \phi_{i}^{\nu} \partial^{\alpha} \phi_{i}^{\beta}-\frac{3 A_{\phi \omega, i}^{\pi^{0}}}{\sqrt{2} F_{\omega_{i}} F_{\phi_{i}}} \pi^{0} \partial^{\mu} \phi_{i}^{\nu} \partial^{\alpha} \omega_{i}^{\beta}\right], \\
& \mathcal{L}_{V V \eta}=-\sum_{i=1}^{n} \frac{4 \sigma_{V_{i}}}{f_{\pi}} \epsilon_{\mu \nu \alpha \beta} \eta\left[\left(\partial^{\mu} \rho_{i}^{\nu} \partial^{\alpha} \rho_{i}^{\beta}+\frac{1}{F_{\omega_{i}}} \partial^{\mu} \omega_{i}^{\nu} \partial^{\alpha} \omega_{i}^{\beta}\right) \frac{1}{2} C_{q}-\frac{9 A_{\phi \omega_{i}}^{\eta}}{F_{\omega_{i}}^{2}} \partial^{\mu} \omega_{i}^{\nu} \partial^{\alpha} \omega_{i}^{\beta}-\frac{1}{F_{\phi_{i}}} \partial^{\mu} \phi_{i}^{\nu} \partial^{\alpha} \phi_{i}^{\beta} \frac{1}{\sqrt{2}} C_{s}\right. \\
& \left.-\frac{9 A_{\phi \omega, i}^{\eta}}{2 F_{\phi_{i}}^{2}} \partial^{\mu} \phi_{i}^{\nu} \partial^{\alpha} \phi_{i}^{\beta}+\frac{A_{i}^{\eta}}{6 F_{\phi_{i}}}\left(\frac{15}{2} C_{q}-\frac{3}{\sqrt{2}} C_{s}\right) \partial^{\mu} \phi_{i}^{\nu} \partial^{\alpha} \phi_{i}^{\beta}-\frac{9 \sqrt{2} A_{\phi \omega, i}^{\eta}}{F_{\omega_{i}} F_{\phi_{i}}} \partial^{\mu} \phi_{i}^{\nu} \partial^{\alpha} \omega_{i}^{\beta}\right], \\
& \mathcal{L}_{V V \eta^{\prime}}=-\sum_{i=1}^{n} \frac{4 \sigma_{V_{i}}}{f_{\pi}} \epsilon_{\mu \nu \alpha \beta} \eta^{\prime}\left[\left(\partial^{\mu} \rho_{i}^{\nu} \partial^{\alpha} \rho_{i}^{\beta}+\frac{1}{F_{\omega_{i}}} \partial^{\mu} \omega_{i}^{\nu} \partial^{\alpha} \omega_{i}^{\beta}\right) \frac{1}{2} C_{q}^{\prime}+\frac{1}{F_{\phi_{i}}} \partial^{\mu} \phi_{i}^{\nu} \partial^{\alpha} \phi_{i}^{\beta} \frac{1}{\sqrt{2}} C_{s}^{\prime}\right. \\
& \left.+\frac{A_{i}^{\eta^{\prime}}}{6 F_{\phi_{i}}}\left(\frac{15}{2} C_{q}^{\prime}+\frac{3}{\sqrt{2}} C_{s}^{\prime}\right) \partial^{\mu} \phi_{i}^{\nu} \partial^{\alpha} \phi_{i}^{\beta}\right],
\end{aligned}
$$


where $n=3, H_{\omega_{i}}, F_{\phi_{i}}=1$ for $i=2,3, A_{\phi \omega, i}^{P} \neq 0$ only for $i=1$, and $P=\pi^{0}, \eta \cdot C_{q}, C_{q}^{\prime}, C_{s}, C_{s}^{\prime}$ are given by the following formulas

$$
\begin{aligned}
& C_{q}=\frac{f_{\pi}}{\sqrt{3} \cos \left(\theta_{8}-\theta_{0}\right)}\left(\frac{1}{f_{8}} \cos \theta_{0}-\frac{1}{f_{0}} \sqrt{2} \sin \theta_{8}\right), \\
& C_{s}=\frac{f_{\pi}}{\sqrt{3} \cos \left(\theta_{8}-\theta_{0}\right)}\left(\frac{1}{f_{8}} \sqrt{2} \cos \theta_{0}+\frac{1}{f_{0}} \sin \theta_{8}\right), \\
& C_{q}^{\prime}=\frac{f_{\pi}}{\sqrt{3} \cos \left(\theta_{8}-\theta_{0}\right)}\left(\frac{1}{f_{0}} \sqrt{2} \cos \theta_{8}+\frac{1}{f_{8}} \sin \theta_{0}\right), \\
& C_{s}^{\prime}=\frac{f_{\pi}}{\sqrt{3} \cos \left(\theta_{8}-\theta_{0}\right)}\left(\frac{1}{f_{0}} \cos \theta_{8}-\frac{1}{f_{8}} \sqrt{2} \sin \theta_{0}\right) .
\end{aligned}
$$

The model from [9] is recovered by setting $n=2, H_{\omega_{i}}=F_{\phi_{i}}=1, A_{i}^{P}=0$, and $A_{\phi \omega, i}^{P}=0$. The couplings in the Lagrangians $\mathcal{L}_{V V P}$ are chosen to fulfil the asymptotic behavior of the $P-\gamma^{*}-\gamma^{*}$ form factors. It is discussed later in this section.

From the Lagrangians, Eqs. (1)-(8), one derives the $P-\gamma^{*}-\gamma^{*}$ amplitude

$$
\mathcal{M}\left[P \rightarrow \gamma^{*}\left(q_{1}\right) \gamma^{*}\left(q_{2}\right)\right]=e^{2} \epsilon_{\mu \nu \alpha \beta} q_{1}^{\mu} q_{2}^{\alpha} F_{\gamma^{*} \gamma^{*} P}\left(t_{1}, t_{2}\right) .
$$

The form factors $F_{\gamma^{*} \gamma^{*} P}\left(t_{1}, t_{2}\right)$ read

$$
\begin{aligned}
& F_{\gamma^{*} \gamma^{*} \pi^{0}}\left(t_{1}, t_{2}\right)=-\frac{N_{c}}{12 \pi^{2} f_{\pi}}+\sum_{i=1}^{n} \frac{4 \sqrt{2} h_{V_{i}} f_{V_{i}}}{3 f_{\pi}} t_{1}\left(D_{\rho_{i}}\left(t_{1}\right)+F_{\omega_{i}} H_{\omega_{i}} D_{\omega_{i}}\left(t_{1}\right)+A_{i}^{\pi_{0}} F_{\phi_{i}} D_{\phi_{i}}\left(t_{1}\right)\right) \\
& +\sum_{i=1}^{n} \frac{4 \sqrt{2} h_{V_{i}} f_{V_{i}}}{3 f_{\pi}} t_{2}\left(D_{\rho_{i}}\left(t_{2}\right)+F_{\omega_{i}} H_{\omega_{i}} D_{\omega_{i}}\left(t_{2}\right)+A_{i}^{\pi_{0}} F_{\phi_{i}} D_{\phi_{i}}\left(t_{2}\right)\right) \\
& -\sum_{i=1}^{n} \frac{4 \sigma_{V_{i}} f_{V_{i}}^{2}}{3 f_{\pi}} t_{1} t_{2}\left(D_{\rho_{i}}\left(t_{2}\right) D_{\omega_{i}}\left(t_{1}\right)+D_{\rho_{i}}\left(t_{1}\right) D_{\omega_{i}}\left(t_{2}\right)+\left(A_{i}^{\pi_{0}} F_{\phi_{i}}-A_{\phi \omega, i}^{\pi^{0}}\right) D_{\phi_{i}}\left(t_{1}\right) D_{\phi_{i}}\left(t_{2}\right)\right. \\
& \left.+\left(F_{\omega_{i}} H_{\omega_{i}}-1-A_{\phi \omega, i}^{\pi^{0}}\right) D_{\omega_{i}}\left(t_{1}\right) D_{\omega_{i}}\left(t_{2}\right)+A_{\phi \omega, i}^{\pi^{0}}\left(D_{\phi_{i}}\left(t_{1}\right) D_{\omega_{i}}\left(t_{2}\right)+D_{\phi_{i}}\left(t_{2}\right) D_{\omega_{i}}\left(t_{1}\right)\right)\right), \\
& F_{\gamma^{*} \gamma^{*} \eta}\left(t_{1}, t_{2}\right)=-\frac{N_{c}}{12 \pi^{2} f_{\pi}}\left(\frac{5}{3} C_{q}-\frac{\sqrt{2}}{3} C_{s}\right)+\sum_{i=1}^{n} \frac{4 \sqrt{2} h_{V_{i}} f_{V_{i}}}{3 f_{\pi}} t_{1}\left(\left(3 C_{q} D_{\rho_{i}}\left(t_{1}\right)+\frac{1}{3} F_{\omega_{i}} C_{q} D_{\omega_{i}}\left(t_{1}\right)-\frac{2 \sqrt{2}}{3} C_{s} F_{\phi_{i}} D_{\phi_{i}}\left(t_{1}\right)\right)\right. \\
& \left.+\left(\frac{5}{3} C_{q}-\frac{\sqrt{2}}{3} C_{s}\right) A_{i}^{\eta} F_{\phi_{i}} D_{\phi_{i}}\left(t_{1}\right)\right)+\sum_{i=1}^{n} \frac{4 \sqrt{2} h_{V_{i}} f_{V_{i}}}{3 f_{\pi}} t_{2}\left(\left(3 C_{q} D_{\rho_{i}}\left(t_{2}\right)+\frac{1}{3} C_{q} F_{\omega_{i}} D_{\omega_{i}}\left(t_{2}\right)\right.\right. \\
& \left.\left.-\frac{2 \sqrt{2}}{3} C_{s} F_{\phi_{i}} D_{\phi_{i}}\left(t_{2}\right)\right)+\left(\frac{5}{3} C_{q}-\frac{\sqrt{2}}{3} C_{s}\right) A_{i}^{\eta} F_{\phi_{i}} D_{\phi_{i}}\left(t_{2}\right)\right)-\sum_{i=1}^{n} \frac{8 \sigma_{V_{i}} f_{V_{i}}^{2}}{f_{\pi}} t_{1} t_{2}\left[\left(\frac{1}{2} C_{q} D_{\rho_{i}}\left(t_{1}\right) D_{\rho_{i}}\left(t_{2}\right)\right.\right. \\
& \left.+\frac{1}{18} F_{\omega_{i}} C_{q} D_{\omega_{i}}\left(t_{1}\right) D_{\omega_{i}}\left(t_{2}\right)-A_{\phi \omega, i}^{\eta} D_{\omega_{i}}\left(t_{1}\right) D_{\omega_{i}}\left(t_{2}\right)-\frac{\sqrt{2}}{9} C_{s} F_{\phi_{i}} D_{\phi_{i}}\left(t_{1}\right) D_{\phi_{i}}\left(t_{2}\right)\right) \\
& \left.+\frac{A_{i}^{\eta} F_{\phi_{i}}}{6}\left(\frac{5}{3} C_{q}-\frac{\sqrt{2}}{3} C_{s}\right) D_{\phi_{i}}\left(t_{1}\right) D_{\phi_{i}}\left(t_{2}\right)-A_{\phi \omega, i}^{\eta} D_{\phi_{i}}\left(t_{1}\right) D_{\phi_{i}}\left(t_{2}\right)+A_{\phi \omega, i}^{\eta}\left(D_{\phi_{i}}\left(t_{1}\right) D_{\omega_{i}}\left(t_{2}\right)+D_{\phi_{i}}\left(t_{2}\right) D_{\omega_{i}}\left(t_{1}\right)\right)\right],
\end{aligned}
$$

and 


$$
\begin{aligned}
F_{\gamma^{*} \gamma^{*} \eta^{\prime}}\left(t_{1}, t_{2}\right)= & -\frac{N_{c}}{12 \pi^{2} f_{\pi}}\left(\frac{5}{3} C_{q}^{\prime}+\frac{\sqrt{2}}{3} C_{s}^{\prime}\right)+\sum_{i=1}^{n} \frac{4 \sqrt{2} h_{V_{i}} f_{V_{i}}}{3 f_{\pi}} t_{1}\left(\left(3 C_{q}^{\prime} D_{\rho_{i}}\left(t_{1}\right)+\frac{1}{3} F_{\omega_{i}} C_{q}^{\prime} D_{\omega_{i}}\left(t_{1}\right)+\frac{2 \sqrt{2}}{3} C_{s}^{\prime} F_{\phi_{i}} D_{\phi_{i}}\left(t_{1}\right)\right)\right. \\
& \left.+\left(\frac{5}{3} C_{q}^{\prime}+\frac{\sqrt{2}}{3} C_{s}^{\prime}\right) A_{i}^{\eta^{\prime}} F_{\phi_{i}} D_{\phi_{i}}\left(t_{1}\right)\right)+\sum_{i=1}^{n} \frac{4 \sqrt{2} h_{V_{i}} f_{V_{i}}}{3 f_{\pi}} t_{2}\left(\left(3 C_{q}^{\prime} D_{\rho_{i}}\left(t_{2}\right)+\frac{1}{3} F_{\omega_{i}} C_{q}^{\prime} D_{\omega_{i}}\left(t_{2}\right)\right.\right. \\
& \left.\left.+\frac{2 \sqrt{2}}{3} C_{s}^{\prime} F_{\phi_{i}} D_{\phi_{i}}\left(t_{2}\right)\right)+\left(\frac{5}{3} C_{q}^{\prime}+\frac{\sqrt{2}}{3} C_{s}^{\prime}\right) A_{i}^{\eta^{\prime}} F_{\phi_{i}} D_{\phi_{i}}\left(t_{2}\right)\right)-\sum_{i=1}^{n} \frac{8 \sigma_{V_{i}} f_{V_{i}}^{2}}{f_{\pi}} t_{1} t_{2}\left[\left(\frac{1}{2} C_{q}^{\prime} D_{\rho_{i}}\left(t_{1}\right) D_{\rho_{i}}\left(t_{2}\right)\right.\right. \\
& \left.+\frac{1}{18} F_{\omega_{i}} C_{q}^{\prime} D_{\omega_{i}}\left(t_{1}\right) D_{\omega_{i}}\left(t_{2}\right)+\frac{\sqrt{2}}{9} C_{s}^{\prime} F_{\phi_{i}} D_{\phi_{i}}\left(t_{1}\right) D_{\phi_{i}}\left(t_{2}\right)\right) \\
& \left.+\frac{A_{i}^{\eta^{\prime}} F_{\phi_{i}}}{6}\left(\frac{5}{3} C_{q}^{\prime}+\frac{\sqrt{2}}{3} C_{s}^{\prime}\right) D_{\phi_{i}}\left(t_{1}\right) D_{\phi_{i}}\left(t_{2}\right)\right]
\end{aligned}
$$

where the vector-meson propagators $D_{V_{i}}\left(Q^{2}\right)$ in the spacelike region are defined by

$$
D_{V_{i}}\left(Q^{2}\right)=\left[Q^{2}-M_{V_{i}}^{2}\right]^{-1} \text {. }
$$

In the timelike region we use the propagators $D_{V_{i}}\left(Q^{2}\right)$ in the following form:

$$
D_{V_{i}}\left(Q^{2}\right)=\left[Q^{2}-M_{V_{i}}^{2}+i \sqrt{Q^{2}} \Gamma_{V_{i}}\right]^{-1} .
$$

We require that the form factors $F_{\gamma^{*} \gamma^{*} P}\left(t_{1}, t_{2}\right)$ vanish, for any value of $t_{2}\left(t_{1}\right)$, when photon virtuality $t_{1}\left(t_{2}\right)$ goes to infinity. This constraint leads to the following relations between the couplings:

$$
\begin{gathered}
-\frac{N_{c}}{4 \pi^{2}}+4 \sqrt{2} \sum_{i=1}^{n} h_{V_{i}} f_{V_{i}}\left(1+F_{\omega_{i}} H_{\omega_{i}}+A_{i}^{\pi^{0}} F_{\phi_{i}}\right)=0 \\
\sqrt{2} h_{V_{i}} f_{V_{i}}-\sigma_{V_{i}} f_{V_{i}}^{2}=0, \quad i=1, \ldots, n \\
-\frac{N_{c}}{4 \pi^{2}}\left(\frac{5}{3} C_{q}-\frac{\sqrt{2}}{3} C_{s}\right)+4 \sqrt{2} \sum_{i=1}^{n} h_{V_{i}} f_{V_{i}}\left[\left(3 C_{q}+\frac{1}{3} F_{\omega_{i}} C_{q}-\frac{2 \sqrt{2}}{3} C_{s} F_{\phi_{i}}\right)+\left(\frac{5}{3} C_{q}-\frac{\sqrt{2}}{3} C_{s}\right) A_{i}^{\eta} F_{\phi_{i}}\right]=0,
\end{gathered}
$$

and

$$
-\frac{N_{c}}{4 \pi^{2}}\left(\frac{5}{3} C_{q}^{\prime}+\frac{\sqrt{2}}{3} C_{s}^{\prime}\right)+4 \sqrt{2} \sum_{i=1}^{n} h_{V_{i}} f_{V_{i}}\left[\left(3 C_{q}^{\prime}+\frac{1}{3} F_{\omega_{i}} C_{q}^{\prime}+\frac{2 \sqrt{2}}{3} C_{s}^{\prime} F_{\phi_{i}}\right)+\left(\frac{5}{3} C_{q}^{\prime}+\frac{\sqrt{2}}{3} C_{s}^{\prime}\right) A_{i}^{\eta^{\prime}} F_{\phi_{i}}\right]=0 .
$$

These relations allow us to determine six of the model parameters. We have chosen $\sigma_{V_{i}} f_{V_{i}}^{2}(i=1,2,3), h_{V_{3}} f_{V_{3}}, A_{2}^{\eta}$, and $A_{2}^{\eta^{\prime}}$ to be determined by using the asymptotic relations equations, (20), (19), (21), and (22), correspondingly. The remaining parameters have been fitted to experimental data. From the Lagrangians equations, (1)-(8), one can derive also the $V-P-\gamma^{*}$ amplitudes

$$
\mathcal{M}\left[V(P) \rightarrow P(V)\left(q_{1}\right) \gamma^{*}\left(q_{2}\right)\right]=e \epsilon_{\mu \nu \beta \alpha} q_{1}^{\nu} q_{2}^{\alpha} F_{V P \gamma^{*}}\left(t_{1}\right),
$$

where $t_{1}=q_{2}^{2}$.

The form factors, given here only for the specific channels used in the fits, have the following form:

$$
F_{\rho \pi^{0} \gamma^{*}}\left(t_{1}\right)=\frac{4 \sqrt{2} h_{V_{1}}}{3 f_{\pi}}\left\{1-t_{1} D_{\omega_{1}}\left(t_{1}\right)\right\},
$$




$$
\begin{gathered}
F_{\omega \pi^{0} \gamma^{*}}\left(t_{1}\right)=\frac{4 \sqrt{2} h_{V_{1}}}{f_{\pi}}\left\{H_{\omega_{1}}-\frac{t_{1}}{F_{\omega_{1}}}\left[D_{\rho_{1}}\left(t_{1}\right)+\left(H_{\omega_{1}} F_{\omega_{1}}-1-A_{\phi \omega, 1}^{\pi^{0}}\right) D_{\omega_{1}}\left(t_{1}\right)+A_{\phi \omega, 1}^{\pi^{0}} D_{\phi_{1}}\left(t_{1}\right)\right]\right\}, \\
F_{\phi \pi^{0} \gamma^{*}}\left(t_{1}\right)=\frac{-4 h_{V_{1}}}{f_{\pi}}\left\{A_{1}^{\pi^{0}}-\frac{A_{\phi \omega, 1}^{\pi^{0}}}{F_{\phi_{1}}} t_{1} D_{\omega_{1}}\left(t_{1}\right)-\left(A_{1}^{\pi^{0}}-\frac{A_{\phi \omega, 1}^{\pi^{0}}}{F_{\phi_{1}}}\right) t_{1} D_{\phi_{1}}\left(t_{1}\right)\right\}, \\
F_{\rho \eta \gamma^{*}}\left(t_{1}\right)=\frac{4 \sqrt{2} h_{V_{1}} C_{q}}{f_{\pi}}\left\{1-t_{1} D_{\rho_{1}}\left(t_{1}\right)\right\}, \\
F_{\phi \eta \gamma^{*}}\left(t_{1}\right)=\frac{4 \sqrt{2} h_{V_{1}}}{3 f_{\pi}}\left\{\left[2 C_{s}-\left(\frac{5}{\sqrt{2}} C_{q}-C_{s}\right) A_{1}^{\eta}\right]\left[1-t_{1} D_{\phi_{1}}\left(t_{1}\right)\right]+\frac{9 \sqrt{2} A_{\phi \omega, 1}^{\eta}}{F_{\phi_{1}}}\left[t_{1} D_{\omega_{1}}\left(t_{1}\right)-t_{1} D_{\phi_{1}}\left(t_{1}\right)\right]\right\}, \\
F_{\omega \eta \gamma^{*}}\left(t_{1}\right)=\frac{4 \sqrt{2} h_{V_{1}}}{3 f_{\pi}}\left\{C_{q}\left(1-t_{1} D_{\omega_{1}}\left(t_{1}\right)\right)+\frac{18 A_{\phi \omega, 1}^{\eta}}{F_{\omega_{1}}}\left(D_{\omega_{1}}\left(t_{1}\right)-D_{\phi_{1}}\left(t_{1}\right)\right)\right\}, \\
F_{\rho \eta^{\prime} \gamma^{*}}\left(t_{1}\right)=\frac{4 \sqrt{2} h_{V_{1}} C_{q}^{\prime}}{f_{\pi}}\left\{1-t_{1} D_{\rho_{1}}\left(t_{1}\right)\right\}, \\
F_{\phi \eta^{\prime} \gamma^{*}}\left(t_{1}\right)=\frac{4 \sqrt{2} h_{V_{1}}}{3 f_{\pi}}\left[-2 C_{s}^{\prime}-\left(\frac{5}{\sqrt{2}} C_{q}^{\prime}+C_{s}^{\prime}\right)=\frac{4 \sqrt{2} h_{V_{1}} C_{q}^{\prime}}{3 f_{\pi}}\left\{1-t_{1} D_{\omega_{1}}\left(t_{1}\right)\right\},\right.
\end{gathered}
$$

\section{FITTING THE MODEL PARAMETERS TO THE EXISTING DATA}

We have fitted the parameters of our model to all existing experimental data, which can be described by the Lagrangians equations, (1)-(8), in the spacelike as well as in the timelike region of the photon virtualities. The data in the spacelike region include measurements of the transition form factors for $\pi^{0}, \eta, \eta^{\prime}$ by the BELLE [42], CELLO [43], and CLEO [44] Collaborations. In our model they are predicted in Eqs. (14)-(16). The data in the timelike region include measurements of the cross sections for the reactions $e^{+} e^{-} \rightarrow \pi^{0}(\eta) \gamma$ by the SND $[45,46]$ and CMD2 [47] Collaborations. The formula for the $e^{+} e^{-} \rightarrow$ $P \gamma$ cross section, where $P$ denotes a pseudoscalar $\left(\pi^{0}, \eta\right.$ or $\left.\eta^{\prime}\right)$, reads

$$
\begin{aligned}
\sigma_{e^{+} e^{-} \rightarrow P \gamma}(s)= & \frac{(4 \pi \alpha)^{3}}{24 \pi s}\left(1-\frac{m_{P}^{2}}{s}\right)\left(\frac{s-m_{P}^{2}}{2 \sqrt{s}}\right)^{2} \\
& \cdot\left|F_{\gamma \gamma^{*} P}(0, s)\right|^{2},
\end{aligned}
$$

where $m_{P}$ is the mass of $P, s$ is the Mandelstam variable, and $F_{\gamma \gamma^{*} P}(0, s)$ is one of the transition form factors defined in Eqs. (14)-(16).

In addition, the timelike form factors measured in threebody decays were used in the fit. The following data sets were included and the model parameters were fitted using the formulas given in brackets: the A2 measurement of a decay $\pi^{0} \rightarrow \gamma e^{+} e^{-}$[48] [Eq. (14)], A2 measurement of a decay $\eta \rightarrow \gamma e^{+} e^{-}$[49] [Eq. (15)], BESIII measurement of a decay $\eta^{\prime} \rightarrow \gamma e^{+} e^{-}$[50] [Eq. (16)], A2 measurement of a decay $\omega \rightarrow \pi^{0} e^{+} e^{-}$[49] [Eq. (25)], KLOE-2 measurement of a decay $\phi \rightarrow \pi^{0} e^{+} e^{-}$[51] [Eq. (26)], and KLOE-2 measurement of a decay $\phi \rightarrow \eta e^{+} e^{-}$[52] [Eq. (29)]. For the A2 measurement of a decay $\eta \rightarrow \pi^{0} \gamma \gamma$ [53] a differential cross section was given. The formula describing it reads

$$
\begin{aligned}
d \Gamma(\eta(q) & \left.\rightarrow \pi^{0}(p) \gamma\left(k_{1}\right) \gamma\left(k_{2}\right)\right) \\
& =\frac{1}{4 m_{\eta}}|M|^{2} d \operatorname{Lips}_{3}\left(q ; p, k_{1}, k_{2}\right),
\end{aligned}
$$

with the amplitude given by

$$
\begin{aligned}
M= & \sum_{i, V}\left(\frac{4 \sqrt{2} e h_{V_{i}}}{3 f_{\pi}}\right)^{2} \epsilon_{\mu \nu \alpha \beta} q^{\nu} k_{1}^{\alpha} \epsilon^{\beta}\left(k_{1}\right) g^{\mu \delta} \\
& \times D_{V_{i}}\left(\left(p+k_{2}\right)^{2}\right) \epsilon_{\delta \sigma \delta^{\prime} \sigma^{\prime}} p^{\sigma} k_{2}^{\delta^{\prime}} \epsilon^{\sigma^{\prime}}\left(k_{2}\right) B_{V_{i}} \\
& +\left(k_{1} \leftrightarrow k_{2}\right)
\end{aligned}
$$

where $B_{\phi_{i}}=-\frac{3}{\sqrt{2}} A_{i}^{\pi^{0}}\left[2 C_{s}-\left(\frac{5}{\sqrt{2}} C_{q}-C_{s}\right) A_{i}^{\eta}\right], B_{\rho_{i}}=3 C_{q}$, $B_{\omega_{i}}=3 H_{\omega_{i}} C_{q}$, and $D_{V_{i}}$ is defined in Eq. (18).

The two-body partial decay widths [54] $P \rightarrow \gamma \gamma V \rightarrow$ $e^{+} e^{-}(V=\rho, \omega, \phi), V \rightarrow \pi^{0} \gamma, V \rightarrow \eta \gamma, \phi \rightarrow \eta^{\prime} \gamma, \eta^{\prime} \rightarrow \rho \gamma$, and $\eta^{\prime} \rightarrow \omega \gamma$ were also used in the fits. In our model they are expressed as 


$$
\begin{gathered}
\Gamma(P \rightarrow \gamma \gamma)=\frac{m_{P}^{3} \pi \alpha^{2}}{4}\left|F_{P \gamma^{*} \gamma^{*}}(0,0)\right|^{2}, \\
\Gamma\left(\rho \rightarrow e^{+} e^{-}\right)=\frac{4 \pi \alpha^{2} M_{\rho} f_{V_{1}}^{2}}{3}, \\
\Gamma\left(\omega \rightarrow e^{+} e^{-}\right)=\frac{4 \pi \alpha^{2} M_{\omega} f_{V_{1}}^{2} F_{\omega_{1}}^{2}}{27}, \\
\Gamma\left(\phi \rightarrow e^{+} e^{-}\right)=\frac{8 \pi \alpha^{2} M_{\phi} f_{V_{1}}^{2} F_{\phi_{1}}^{2}}{27}, \\
\Gamma(P \rightarrow V \gamma)=\frac{\alpha}{8} m_{P}^{3} k_{V}^{3}\left|F_{V P \gamma^{*}}(0)\right|^{2}, \\
\Gamma(V \rightarrow P \gamma)=\frac{\alpha}{24} M_{V}^{3} k_{\mathcal{P}}^{3}\left|F_{V P \gamma^{*}}(0)\right|^{2},
\end{gathered}
$$

where $k_{V}=\left(1-\frac{m_{P}^{2}}{M_{V}^{2}}\right), k_{P}=\left(1-\frac{M_{V}^{2}}{m_{P}^{2}}\right)$. The form factors $F_{P \gamma^{*} \gamma^{*}}$ are given in Eqs. (14)-(16) and the form factors $F_{V P \gamma^{*}}$ are given in Eqs. (24)-(31).

We have performed two fits: one with fixed parameters $\theta_{8}, \theta_{0}, f_{8}, f_{0}$, and $f_{\pi}$ describing the $\eta-\eta^{\prime}$ mixing and $\pi^{0} \rightarrow \gamma \gamma$ decay width (called fit 1) and the second one where we fit also these parameters (called fit 2). The $\chi^{2}$ values for all the experimental sets of data obtained in the fits are given in Table I. The BABAR measurement of the $\pi^{0}$ transition form factor [55] as well as the NA60 measurements [56] of the $\eta$ transition form factor and the $F_{\omega \pi^{0} \gamma^{*}}$ form factor were not used in the fits summarized here. They are in contradiction with other experimental data (see Figs. 1, 5, and 6). The smallest tension is between the $\eta$ transition form factor measurements of A2 [49] and NA60 [56] (see Fig. 5) and in fact the data are consistent within the experimental error bars. Yet within the model we developed here there is no way to fit simultaneously SND [45] data on the $e^{+} e^{-} \rightarrow \eta \gamma$ cross section, the differential width $\left(\eta \rightarrow \pi^{0} \gamma \gamma\right)$ measured by A2 [53], and the partial widths $V \rightarrow \eta \gamma$ [54] together with the NA60 measurements [56] of the $\eta$ transition form factor in the timelike region.

In Table II we give the parameters obtained in both fits. The fit is much better if we allow for the changing of the $\eta-\eta^{\prime}$ mixing parameters. In principle one can think of "fit 2 " as a way to extract the $\eta-\eta^{\prime}$ mixing parameters. Yet, one has to remember that this is a model dependent extraction.

To show how the fits represent data for individual data points we present here the following plots:

(i) In Fig. 1 the pseudoscalars' transition form factors in

\begin{tabular}{|c|c|c|c|c|c|c|c|}
\hline Experiment & nep & $\chi^{2}$, Fit 1 & $\chi^{2}$, Fit 2 & Experiment & nep & $\chi^{2}$, Fit 1 & $\chi^{2}$, Fit 2 \\
\hline \multicolumn{8}{|l|}{ Spacelike form factors } \\
\hline BELLE $\left(\pi^{0}\right)$ [42] & 15 & 9.96 & 6.72 & CLEO98 $(\eta)$ [44] & 19 & 15.8 & 15.5 \\
\hline CELLO91 $\left(\pi^{0}\right)$ [43] & 5 & 0.34 & 0.24 & $B A B A R\left(\eta^{\prime}\right)[57]$ & 11 & 5.4 & 3.70 \\
\hline CLEO98 $\left(\pi^{0}\right)[44]$ & 15 & 10.6 & 6.82 & CELLO91 $\left(\eta^{\prime}\right)[43]$ & 5 & 0.73 & 0.56 \\
\hline$B A B A R(\eta)[57]$ & 11 & 7.34 & 7.5 & CLEO98 $\left(\eta^{\prime}\right)[44]$ & 29 & 25.1 & 24.4 \\
\hline CELLO91 $(\eta)[43]$ & 4 & 0.16 & 0.16 & & & & \\
\hline \multicolumn{8}{|l|}{$e^{+} e^{-}$cross sections } \\
\hline $\operatorname{CMD} 2\left(\pi^{0} \gamma\right)[47]$ & 46 & 54.1 & 54.1 & $\operatorname{SND}(\eta \gamma)[45]$ & 78 & 68.7 & 59.8 \\
\hline SND $\left(\pi^{0} \gamma\right)[46]$ & 62 & 65.5 & 54.2 & $B A B A R\left(\eta \gamma, \eta^{\prime} \gamma\right)[58]$ & 2 & 0.18 & 1.57 \\
\hline CMD2 $(\eta \gamma)[47]$ & 42 & 25.4 & 25.6 & & & & \\
\hline \multicolumn{8}{|l|}{ Three-body decays } \\
\hline A2 $\left(\pi^{0} \rightarrow \gamma e^{+} e^{-}\right)[48]$ & 18 & 0.32 & 0.34 & A2 $\left(\omega \rightarrow \pi^{0} e^{+} e^{-}\right)[49]$ & 14 & 2.14 & 2.12 \\
\hline $\mathrm{A} 2\left(\eta \rightarrow \gamma e^{+} e^{-}\right)[49]$ & 34 & 10.2 & 11.1 & $\operatorname{KLOE-2}\left(\phi \rightarrow \pi^{0} e^{+} e^{-}\right)[51]$ & 15 & 4.33 & 4.33 \\
\hline A2 $\left(\eta \rightarrow \pi^{0} \gamma \gamma\right)$ [53] & 7 & 26.6 & 19.5 & KLOE-2 $\left(\phi \rightarrow \eta e^{+} e^{-}\right)[52]$ & 92 & 95.1 & 95.1 \\
\hline $\operatorname{BESIII~}\left(\eta^{\prime} \rightarrow \gamma e^{+} e^{-}\right)[50]$ & 8 & 2.39 & 2.13 & & & & \\
\hline \multicolumn{8}{|l|}{ Two-body decays } \\
\hline$\Gamma\left(\pi^{0} \rightarrow \gamma \gamma\right)$ & 1 & 0.36 & 0.1 & $\Gamma\left(\rho \rightarrow \pi^{0} \gamma\right)$ & 1 & 1.17 & 0.42 \\
\hline$\Gamma(\eta \rightarrow \gamma \gamma)[54]$ & 1 & 0.78 & 2.73 & $\Gamma\left(\omega \rightarrow \pi^{0} \gamma\right)[54]$ & 1 & 4.08 & 1.56 \\
\hline$\Gamma\left(\eta^{\prime} \rightarrow \gamma \gamma\right)[54]$ & 1 & 1.05 & 0.44 & $\Gamma\left(\phi \rightarrow \pi^{0} \gamma\right)$ & 1 & 0.08 & 0.06 \\
\hline$\Gamma\left(\eta^{\prime} \rightarrow \rho \gamma\right)$ & 1 & 3.0 & 0.77 & $\Gamma(\rho \rightarrow \eta \gamma)[54]$ & 1 & 3.32 & 6.8 \\
\hline$\Gamma\left(\eta^{\prime} \rightarrow \omega \gamma\right)$ & 1 & 0.00 & 0.54 & $\Gamma(\omega \rightarrow \eta \gamma)[54]$ & 1 & 6.86 & 3.04 \\
\hline$\Gamma\left(\rho \rightarrow e^{+} e^{-}\right)[54]$ & 1 & 0.23 & 0.05 & $\Gamma(\phi \rightarrow \eta \gamma)[54]$ & 1 & 1.63 & 1.17 \\
\hline$\Gamma\left(\omega \rightarrow e^{+} e^{-}\right)[54]$ & 1 & 0.56 & 0.73 & $\Gamma\left(\phi \rightarrow \eta^{\prime} \gamma\right)$ & 1 & 0.01 & 0.00 \\
\hline \multirow[t]{2}{*}{$\Gamma\left(\phi \rightarrow e^{+} e^{-}\right)[54]$} & 1 & 0.69 & 0.46 & & & & \\
\hline & & & & Total & 536 & 454 & 415 \\
\hline
\end{tabular}
the spacelike region are presented. The "old fit" refers there to the two-octet model from [9]. On the right-hand side of the plots the asymptotic values of the form factors are given within the current model

TABLE I. The values of the $\chi^{2}$ for the experiments used in the fits described in the text. "nep" means number of experimental points. 

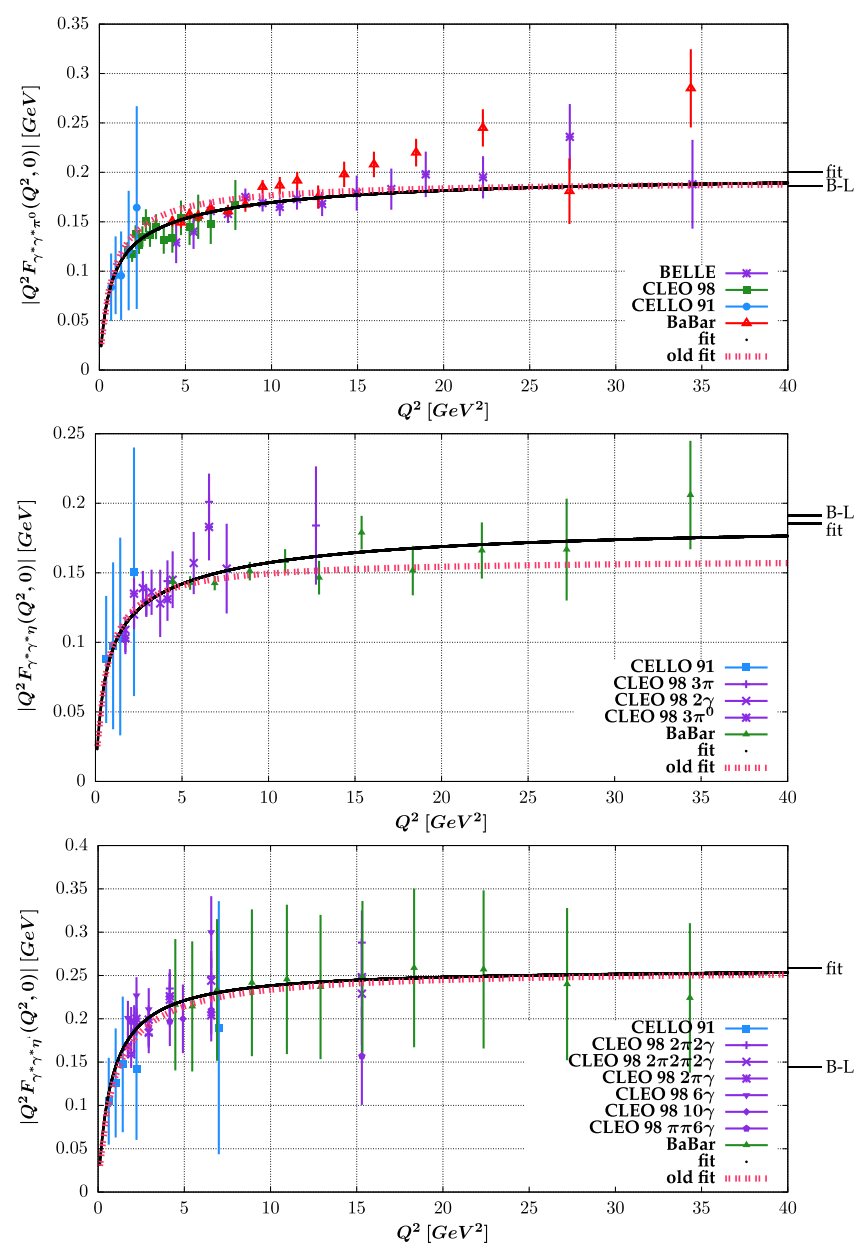

FIG. 1. Transition form factors $\gamma^{*} \gamma P$ in the spacelike region compared to the data.

(fit 2) (see also the discussion in Sec. IV) and as in the original Brodsky-Lapage paper [59], i.e., $2 f_{\pi}$ for the pion form factor, $2 f_{\eta}=2 f_{\pi} /\left(\frac{5}{3} C_{q}-\frac{\sqrt{2}}{3} C_{s}\right)$ for the eta form factor, and $2 f_{\eta^{\prime}}=2 f_{\pi} /\left(\frac{5}{3} C_{q}^{\prime}+\frac{\sqrt{2}}{3} C_{s}^{\prime}\right)$ for the eta prime form factor.

(ii) In Figs. 2-4 the cross sections of the reactions $e^{+} e^{-} \rightarrow \pi^{0} \gamma$ and $e^{+} e^{-} \rightarrow \eta \gamma$ are shown. We show all the data points and fits in Fig. 2 and separately show the regions around $\omega$ (Fig. 3) and $\phi$ (Fig. 4) resonances.

(iii) In Fig. 5 the pseudoscalars' transition form factors in the timelike region are presented.

(iv) In Figs. 6 and 7 the $V P \gamma$ form factors are shown.

(v) In Fig. 8 the differential decay width of $\eta \rightarrow \pi^{0} \gamma \gamma$ decay is presented.

We show only the plots using the parameters from fit 2 . The plots with the fit 1 parameters look similar.

The modeling of the form of the propagators is beyond the scope of this paper as it would require us to go beyond the leading order contributions. Yet, to investigate a possible dependence of the obtained results on the ansatz
TABLE II. Model parameters obtained in the fits. The errors, given in brackets, are the parabolic errors calculated by Minos of the Minuit package. $(f)$ means that the parameter was fixed in the fit to the value given in this table.

\begin{tabular}{lcc}
\hline \hline Parameter & Fit 1 & Fit 2 \\
\hline$h_{V_{1}}$ & $0.0335(2)$ & $0.0377(8)$ \\
$f_{V_{1}}$ & $0.2022(8)$ & $0.2020(8)$ \\
$f_{V_{2}} h_{V_{2}}$ & $-0.0013(2)$ & $-0.0010(4)$ \\
$h_{V_{2}}$ & $0.00184(5)$ & $0.0002(1)$ \\
$h_{V_{3}}$ & $-0.485(7)$ & $-0.30(4)$ \\
$H_{\omega_{1}}$ & $1.160(11)$ & $1.02(3)$ \\
$F_{\omega_{1}}$ & $0.881(8)$ & $0.88(1)$ \\
$F_{\phi_{1}}$ & $0.783(5)$ & $0.783(5)$ \\
$A_{1}^{\pi^{0}}$ & $-0.094(1)$ & $-0.083(2)$ \\
$A_{2}^{\pi^{0}}$ & $-12.04(16)$ & $-15(6)$ \\
$A_{3}^{\pi^{0}}$ & $0.08(3)$ & $-0.16(7)$ \\
$A_{1}^{\eta}$ & $-0.041(4)$ & $-0.30(4)$ \\
$A_{3,}^{\eta}$ & $0.23(6)$ & $-0.06(8)$ \\
$A_{1}^{\eta^{\prime}}$ & $-0.039(7)$ & $-0.21(5)$ \\
$A_{3}^{\eta^{\prime}}$ & $-0.27(3)$ & $-0.56(6)$ \\
$A_{\phi \omega, 1}^{\pi^{0}}$ & $-0.23(4)$ & $-0.21(4)$ \\
$A_{\phi \omega, 1}^{\eta}$ & $-0.031(8)$ & $-0.028(7)$ \\
$f_{\pi}$ & $0.092388(f)$ & $0.09266(8)$ \\
$f_{0}$ & $0.10623(f)$ & $0.095(2)$ \\
$f_{8}$ & $0.11697(f)$ & $0.17(1)$ \\
$\theta_{0}$ & $-0.14471(f)$ & $-0.54(12)$ \\
$\theta_{8}$ & $-0.36516(f)$ & $-0.446(17)$ \\
\hline \hline
\end{tabular}

given in Eqs. (17) and (18), we have performed the fits with constant imaginary parts, replacing $\sqrt{Q^{2}}$ in Eq. (18) with $M_{V_{i}}$. There is only a small difference between the fits. For fit 1 (fixed $\eta-\eta^{\prime}$ mixing) we get $\chi^{2}=469$, while for fit 2
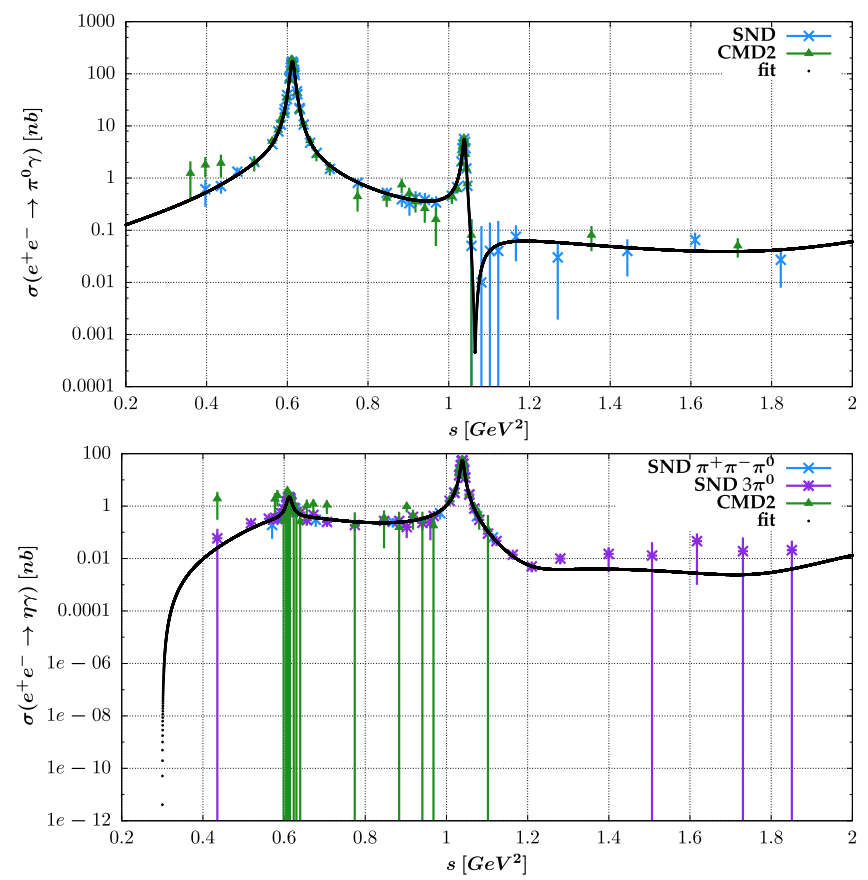

FIG. 2. Experimental data for $\sigma\left(e^{+} e^{-} \rightarrow P \gamma\right)$ compared to the model predictions. 

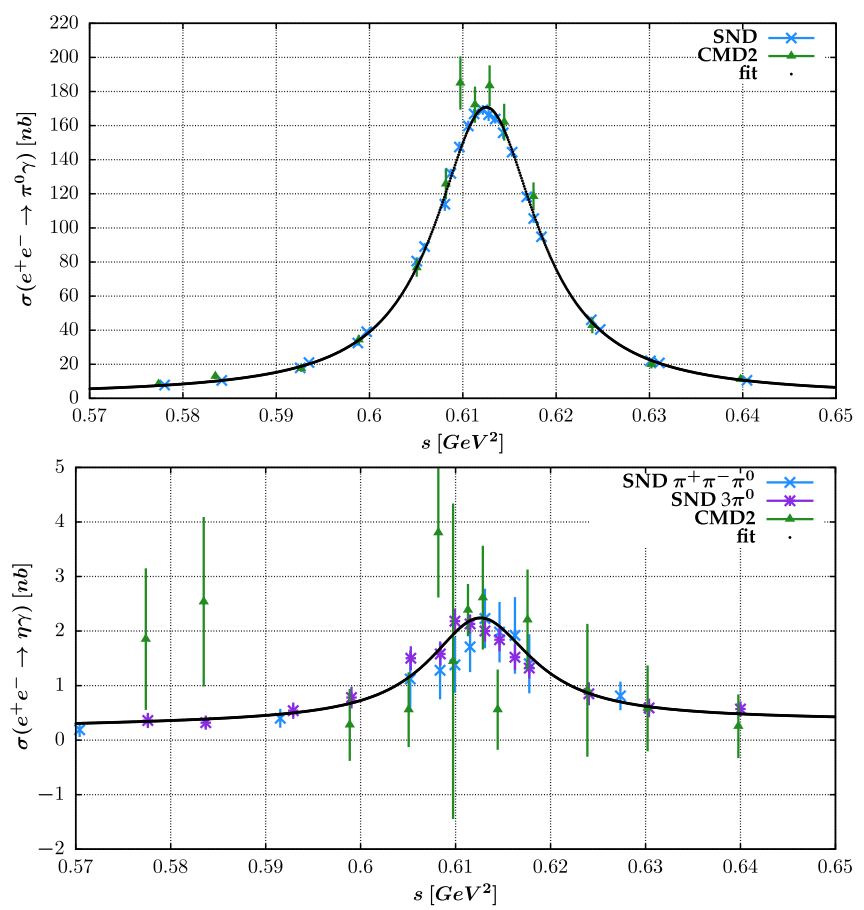

FIG. 3. Experimental data for $\sigma\left(e^{+} e^{-} \rightarrow P \gamma\right)$ compared to the model predictions. The region of the $s$ has been limited to the $\omega$ peak.
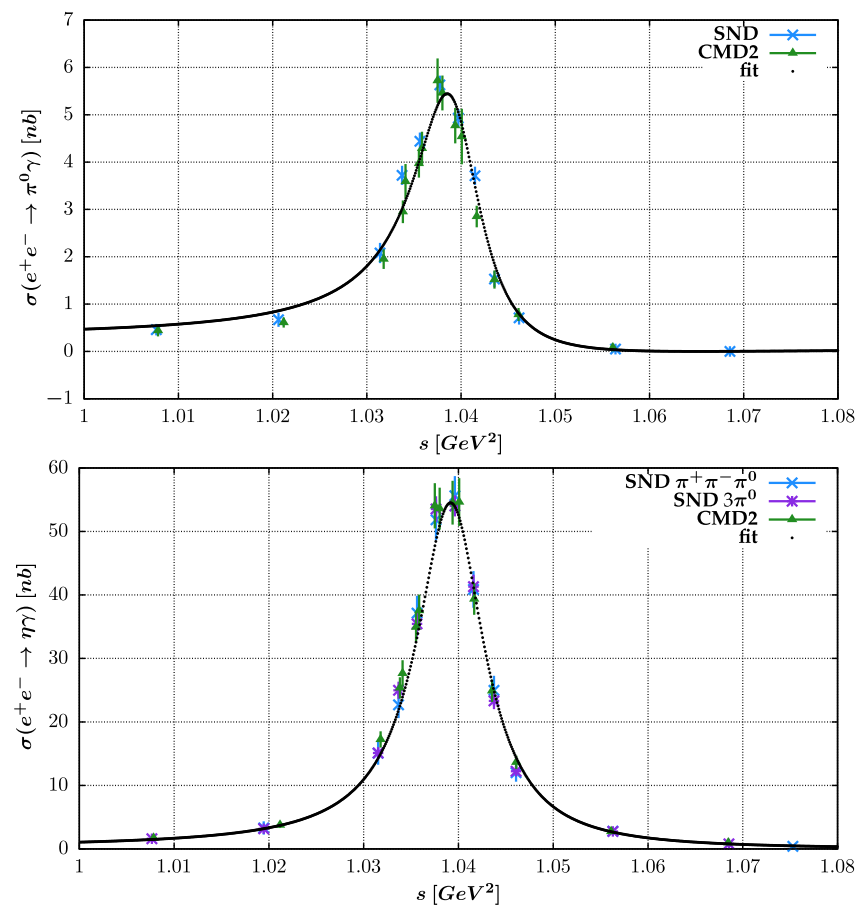

FIG. 4. Experimental data for $\sigma\left(e^{+} e^{-} \rightarrow P \gamma\right)$ compared to the model predictions. The region of the $s$ has been limited to the $\phi$ peak.

(with fitted $\eta-\eta^{\prime}$ mixing parameters) we get $\chi^{2}=409$. There are small changes of $\chi^{2}$ in all data sets. Moreover, the results for $a_{\mu}$ (see Sec. V) are in agreement, within the parametric errors, between the version of the model with
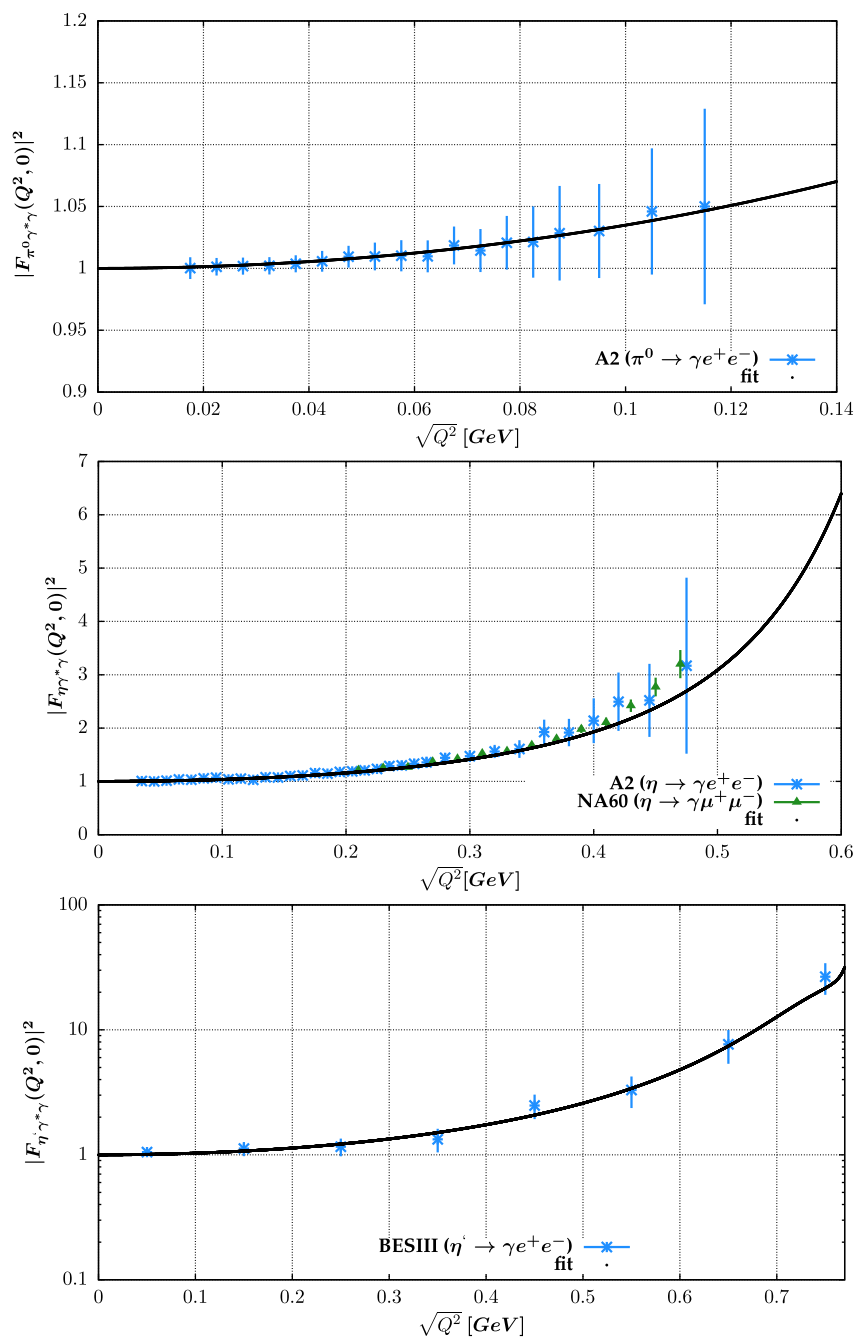

FIG. 5. Transition form factor $\gamma^{*} \gamma P$ in the timelike region compared to the data.

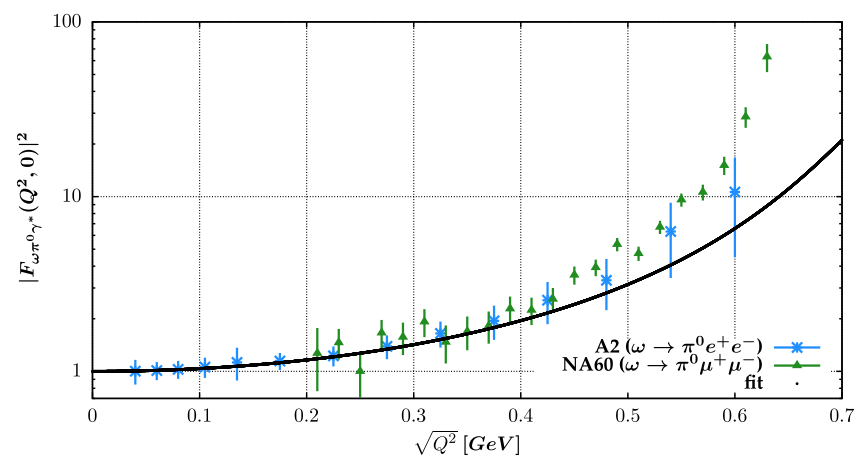

FIG. 6. The form factor $\omega \pi^{0} \gamma$ in the timelike region compared to the data.

the propagators given by Eq. (18) and the version, where one uses constant widths. However not all ad hoc choices are allowed as some of them alter the leading asymptotic behavior of the form factors. This is the case, if one adopts 

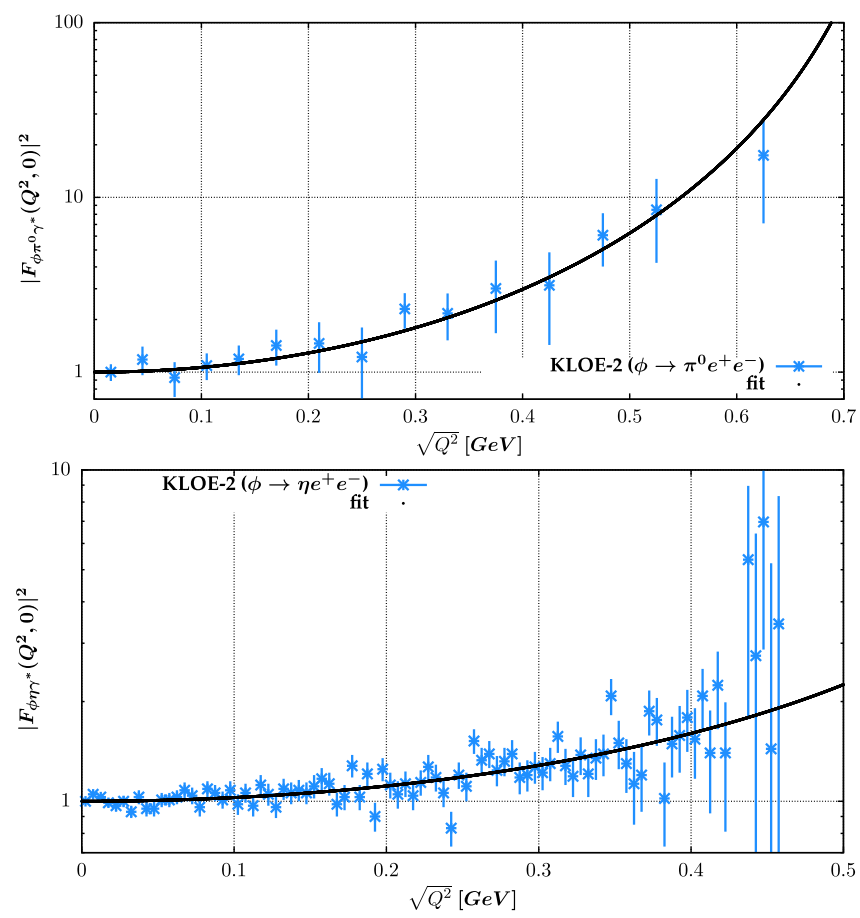

FIG. 7. The form factor $\phi P \gamma$ in the timelike region compared to the data.

the imaginary part from the Gounaris-Sakurai parametrization of the $\rho$ propagator [60], "forgetting" its real part coming from the pion loop integral. The imaginary part of the denominator is then $\sim Q^{2}$ and alters the leading asymptotic behavior in the timelike region, leaving the one in the spacelike region unchanged. These kinds of ad hoc choices do not fit to the data as they predict different asymptotic behavior at large timelike and spacelike

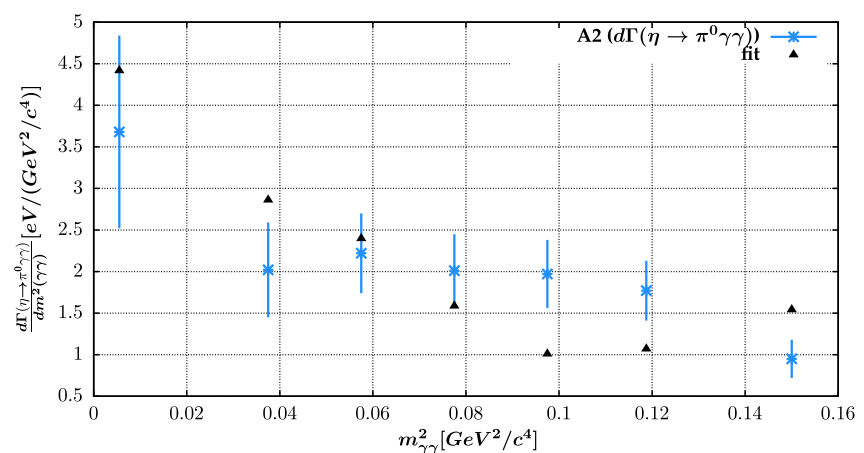

FIG. 8. The differential partial width of the decay $\eta \rightarrow \pi^{0} \gamma \gamma$ compared to the data.

invariants, in clear contradiction to the experimental data [58].

\section{THE ASYMPTOTICS OF THE FORM FACTORS AND THE SLOPE OF THE FORM FACTORS AT THE ORIGIN}

The analytic form of the asymptotic behavior of the form factors is analogous to the one obtained in [9] with the asymptotic limits changed. As discussed in [61], it is difficult to incorporate all the short distance constraints coming from QCD into a model, where only hadron interactions are considered. The class of models we investigate in this paper is not an exception. The asymptotic behavior of the form factors for two equal invariants predicted in QCD to be $F_{\gamma^{*} \gamma^{*} P}(t, t) \sim 1 / t$ [62] is instead $F_{\gamma^{*} \gamma^{*} P}(t, t) \sim 1 / t^{2}$. For completeness we report here the formulas, but skip the detailed discussion as it should repeat the one presented in [9]. They read

$$
\begin{gathered}
F_{\gamma^{*} \gamma \pi^{0}}(t, 0)=\sum_{i=1}^{n} \frac{4 \sqrt{2} h_{V_{i}} f_{V_{i}}}{3 f_{\pi}} \frac{1}{t}\left(M_{\rho_{i}}^{2}+F_{\omega_{i}} H_{\omega_{i}} M_{\omega_{i}}^{2}+A_{i}^{\pi^{0}} F_{\phi_{i}} M_{\phi_{i}}^{2}\right)+O\left(\frac{1}{t^{2}}\right) \\
F_{\gamma^{*} \gamma^{*} \pi^{0}}(t, t)=\sum_{i=1}^{n} \frac{4 \sqrt{2} h_{V_{i}} f_{V_{i}}}{3 f_{\pi}} \frac{1}{t^{2}}\left(-2 M_{\rho_{i}}^{2} M_{\omega_{i}}^{2}-\left(A_{i}^{\pi^{0}} F_{\phi_{i}}-A_{\phi \omega, i}^{\pi^{0}}\right) M_{\phi_{i}}^{4}-2 A_{\phi \omega, i}^{\pi^{0}} M_{\phi_{i}}^{2} M_{\omega_{i}}^{2}-\left(F_{\omega_{i}} H_{\omega_{i}}-1-A_{\phi \omega, i}^{\pi^{0}}\right) M_{\omega_{i}}^{4}\right)+O\left(\frac{1}{t^{3}}\right), \\
F_{\gamma^{*} \gamma \eta}(t, 0)=\sum_{i=1}^{n} \frac{4 \sqrt{2} h_{V_{i}} f_{V_{i}}}{3 f_{\pi}} \frac{1}{t}\left(3 C_{q} M_{\rho_{i}}^{2}+\frac{1}{3} F_{\omega_{i}} C_{q} M_{\omega_{i}}^{2}-\frac{2 \sqrt{2}}{3} C_{s} F_{\phi_{i}} M_{\phi_{i}}^{2}+\left(\frac{5}{3} C_{q}-\frac{\sqrt{2}}{3} C_{s}\right) A_{i}^{\eta} F_{\phi_{i}} M_{\phi_{i}}^{2}\right)+O\left(\frac{1}{t^{2}}\right) \\
F_{\gamma^{*} \gamma^{*} \eta}(t, t)=\sum_{i=1}^{n} \frac{8 \sqrt{2} h_{V_{i}} f_{V_{i}}}{f_{\pi}^{2}}\left(-\frac{1}{2} C_{q} M_{\rho_{i}}^{4}-\frac{1}{18} F_{\omega_{i}} C_{q} M_{\omega_{i}}^{4}+A_{\phi \omega, i}^{\eta} M_{\omega_{i}}^{4}+\frac{\sqrt{2}}{9} C_{s} F_{\phi_{i}} M_{\phi_{i}}^{4}\right. \\
\left.-\frac{A_{i}^{\eta} F_{\phi_{i}}}{6}\left(\frac{5}{3} C_{q}-\frac{\sqrt{2}}{3} C_{s}\right) M_{\phi_{i}}^{4}+A_{\phi \omega, i}^{\eta} M_{\phi_{i}}^{4}+2 A_{\phi \omega, i}^{\eta} M_{\phi_{i}}^{2} M_{\omega_{i}}^{2}\right)+O\left(\frac{1}{t^{3}}\right)
\end{gathered}
$$


$F_{\gamma^{*} \gamma \eta^{\prime}}(t, 0)=\sum_{i=1}^{n} \frac{4 \sqrt{2} h_{V_{i}} f_{V_{i}}}{3 f_{\pi}} \frac{1}{t}\left(3 C_{q}^{\prime} M_{\rho_{i}}^{2}+\frac{1}{3} F_{\omega_{i}} C_{q}^{\prime} M_{\omega_{i}}^{2}+\frac{2 \sqrt{2}}{3} C_{s}^{\prime} F_{\phi_{i}} M_{\phi_{i}}^{2}+\left(\frac{5}{3} C_{q}^{\prime}+\frac{\sqrt{2}}{3} C_{s}^{\prime}\right) A_{i}^{\eta^{\prime}} F_{\phi_{i}} M_{\phi_{i}}^{2}\right)+O\left(\frac{1}{t^{2}}\right)$,

$F_{\gamma^{*} \gamma^{*} \eta^{\prime}}(t, t)=\sum_{i=1}^{n} \frac{-8 \sqrt{2} h_{V_{i}} f_{V_{i}}}{f_{\pi}} \frac{1}{t^{2}}\left(\frac{1}{2} C_{q}^{\prime} M_{\rho_{i}}^{4}+\frac{1}{18} F_{\omega_{i}} C_{q}^{\prime} M_{\omega_{i}}^{4}+\frac{\sqrt{2}}{9} C_{s}^{\prime} F_{\phi_{i}} M_{\phi_{i}}^{4}+\frac{A_{i}^{\eta^{\prime}} F_{\phi_{i}}}{6}\left(\frac{5}{3} C_{q}^{\prime}+\frac{\sqrt{2}}{3} C_{s}^{\prime}\right) M_{\phi_{i}}^{4}\right)+O\left(\frac{1}{t^{3}}\right)$.

The models are compared often by comparing the slopes of the form factors at the origin which we denote as $a_{P}$. For the pseudoscalar transition form factors, they are defined as

$$
\left.a_{P} \equiv \frac{1}{F_{\gamma^{*} \gamma^{*} P}(0,0)} \frac{d F_{\gamma^{*} \gamma^{*} P}(t, 0)}{d x}\right|_{t=0}
$$

where $x \equiv \frac{t}{m_{P}^{2}}$. The model predictions for the model developed in this paper read

$$
\begin{gathered}
a_{\pi^{0}}=\frac{16 \sqrt{2} \pi^{2} m_{\pi^{0}}^{2}}{N_{c}} \sum_{i=1}^{3} h_{V_{i}} f_{V_{i}}\left(\frac{1}{M_{\rho_{i}}^{2}}+F_{\omega_{i}} H_{\omega_{i}} \frac{1}{M_{\omega_{i}}^{2}}+A_{i}^{\pi^{0}} F_{\phi_{i}} \frac{1}{M_{\phi_{i}}^{2}}\right) \\
a_{\eta}=\frac{16 \sqrt{2} \pi^{2} m_{\eta}^{2}}{N_{c}\left(\frac{5}{3} C_{q}-\frac{\sqrt{2}}{3} C_{s}\right)} \sum_{i=1}^{3} h_{V_{i}} f_{V_{i}}\left(3 C_{q} \frac{1}{M_{\rho_{i}}^{2}}+\frac{1}{3} F_{\omega_{i}} C_{q} \frac{1}{M_{\omega_{i}}^{2}}-\frac{2 \sqrt{2}}{3} C_{s} F_{\phi_{i}} \frac{1}{M_{\phi_{i}}^{2}}+\left(\frac{5}{3} C_{q}-\frac{\sqrt{2}}{3} C_{s}\right) A_{i}^{\eta} F_{\phi_{i}} \frac{1}{M_{\phi_{i}}^{2}}\right) \\
a_{\eta^{\prime}}=\frac{16 \sqrt{2} \pi^{2} m_{\eta^{\prime}}^{2}}{N_{c}\left(\frac{5}{3} C_{q}^{\prime}+\frac{\sqrt{2}}{3} C_{s}^{\prime}\right)} \sum_{i=1}^{3} h_{V_{i}} f_{V_{i}}\left(3 C_{q}^{\prime} \frac{1}{M_{\rho_{i}}^{2}}+\frac{1}{3} F_{\omega_{i}} C_{q}^{\prime} \frac{1}{M_{\omega_{i}}^{2}}+\frac{2 \sqrt{2}}{3} C_{s}^{\prime} F_{\phi_{i}} \frac{1}{M_{\phi_{i}}^{2}}+\left(\frac{5}{3} C_{q}^{\prime}+\frac{\sqrt{2}}{3} C_{s}^{\prime}\right) A_{i}^{\eta^{\prime}} F_{\phi_{i}} \frac{1}{M_{\phi_{i}}^{2}}\right) .
\end{gathered}
$$

The numerical comparison between predictions within different models and direct extractions from recent experiments is made in Table III. The obtained results are in fair agreement with both.

TABLE III. The slope parameter $a_{P}$ [Eq. (47)] compared to other model predictions and experimental data.

\begin{tabular}{lccc}
\hline \hline Model & $a_{\pi^{0}}$ & $a_{\eta}$ & $a_{\eta^{\prime}}$ \\
\hline Fit 1 & $0.0298(3)$ & $0.542(4)$ & $1.357(9)$ \\
Fit 2 & $0.0310(7)$ & $0.536(11)$ & $1.39(3)$ \\
{$[9]$} & $0.02870(9)$ & $0.521(2)$ & $1.323(4)$ \\
{$[63]$} & 0.0324 & 0.506 & 1.470 \\
{$[64]$} & $\ldots$ & $0.62+0.06-0.03$ & $\ldots$ \\
{$[27]$} & $\ldots$ & $0.60(6)_{s t}(3)_{s y}$ & $1.30(15)_{s t}(7)_{s y}$ \\
{$[29]$} & $\ldots$ & $0.576(11)_{s t}(4)_{s y}$ & $\ldots$ \\
{$[30]$} & $\ldots$ & $\ldots .428(63)$ & $1.31(4)$ \\
CELLO [43] & $0.0326(26)$ & $\ldots$ & $\ldots$ \\
SINDRUM-I & $0.026(24)_{s t}(48)_{s y}$ & $\ldots$ & $\ldots$ \\
[65] & & $\ldots$ & $\ldots$ \\
[66] & $0.025(14)_{s t}(26)_{s y}$ & $0.576(105)_{s t}(39)_{s y}$ & $\ldots$ \\
Mami [67] & $\ldots$ & $0.585(18)_{s t}(13)_{s y}$ & $\ldots$ \\
NA60 [68] & $\ldots$ & $\ldots$ & $\ldots$ \\
NA62 [69] & $0.0368(51)_{s t}(25)_{s y}$ & &
\end{tabular}

\section{PSEUDOSCALAR CONTRIBUTIONS TO $a_{\mu}$}

Within the model described in the previous sections, we calculate the contributions from the pseudoscalar mesons $\pi^{0}, \eta$, and $\eta^{\prime}$ to the muon anomalous magnetic moment $a_{\mu}$. Equation (155) of [10] was used with the form factors developed in this paper, Eqs. (14)-(16). The variables spanned from zero to infinity were mapped on the intervals $(0,1)$ and the integrals were performed using the Monte Carlo method. For a cross check of the numerical method and the implementation we have recovered values from Table 7 of [10] using the model(s) presented there. The results are presented in Table IV for both fits and compared with previous calculations. For the error evaluation we have used the covariance matrix calculated by Minuit from CERNLIB. The derivatives of the $a_{\mu}$ with respect to the fitting parameters were calculated numerically, using the Monte Carlo method to obtain the necessary integrals. The error of the sum of all the contributions from pseudoscalars was calculated separately as an error on the function being the sum of the free contributions. As one can 
TABLE IV. Pseudoscalar-exchange contribution to the $a_{\mu}^{H L B L, P S} \times 10^{11}\left(P S=\pi^{0}, \eta, \eta^{\prime}\right)$.

\begin{tabular}{lcccc}
\hline \hline Model & $a_{\mu}^{\pi^{0}}$ & $a_{\mu}^{\eta}$ & $a_{\mu}^{\eta^{\prime}}$ & $a_{\mu}^{P}$ \\
\hline Fit 1 & $58.80 \pm 0.27$ & $13.56 \pm 0.10$ & $12.97 \pm 0.09$ & $85.32 \pm 0.30$ \\
Fit 2 & $56.96 \pm 0.94$ & $13.35 \pm 0.45$ & $12.55 \pm 0.48$ & $82.85 \pm 1.15$ \\
Fit 3 & $59.07 \pm 0.17$ & $13.52 \pm 0.09$ & $12.96 \pm 0.09$ & $85.55 \pm 0.22$ \\
Fit 4 & $57.79 \pm 0.90$ & $13.31 \pm 0.19$ & $12.31 \pm 0.21$ & $83.41 \pm 0.94$ \\
{$[74]$} & $57.4 \pm 6.0$ & $13.4 \pm 1.6$ & $11.9 \pm 1.4$ & $82.7 \pm 6.4$ \\
{$[75]$} & $58 \pm 10$ & $13 \pm 1$ & $12 \pm 1$ & $83 \pm 12$ \\
{$[76]$} & $\cdots$ & $\cdots$ & $\cdots$ & $85 \pm 13$ \\
{$[70]$} & $76.5 \pm 6.5$ & $18 \pm 1.4$ & $18 \pm 1.5$ & $114 \pm 10$ \\
{$[77]$} & $62.7-66.8$ & $\cdots$ & $\cdots$ & $\cdots$ \\
{$[10,78]$} & $72 \pm 12$ & $14.5 \pm 4.8$ & $12.5 \pm 4.2$ & $99 \pm 16$ \\
{$[71]$} & $68.8 \pm 1.2$ & $\cdots$ & $\cdots$ & $\cdots$ \\
{$[72]$} & $66.6 \pm 2.1$ & $20.4 \pm 4.4$ & $17.7 \pm 2.3$ & $104.7 \pm 5.4$ \\
{$[79]$} & $65.0 \pm 8.3$ & $\cdots$ & $\cdots$ & $\cdots$ \\
\hline \hline
\end{tabular}

observe, the obtained results are consistent with most of the other models. The biggest differences, not contained in the error bars, are observed with calculations presented in [70-72]. The much smaller errors of our calculations, as compared to other results, are only parametric and do not cover the model dependence. Yet, it has to be stressed that the model is able to describe well all the existent data on the form factors both in the spacelike and timelike regions. To cover the model dependence within the class of models we consider here we added two values of $a_{\mu}$ (fit 4 and fit 5). In the models 4 and 5 we have excluded from the fit the cross sections of the reactions $e^{+} e^{-} \rightarrow \eta \gamma$ and $e^{+} e^{-} \rightarrow \eta^{\prime} \gamma$ measured by $B A B A R$ [58] at very high energy compared to other data points. The fits were performed with parameters $A_{3}^{P}$ set to zero and with fixed or fitted mixing parameters similarly to fits 1 and 2 . The $e^{+} e^{-} \rightarrow \eta^{\prime} \gamma$ calculated at the BABAR energy point is off the measured value by about 5 standard deviations. Also the predicted $e^{+} e^{-} \rightarrow \pi^{0} \gamma$ cross section at $s=112 \mathrm{GeV}^{2}$ is different for both fits. However, as expected from the analysis in [73], the values or the pseudoscalar form factors at large invariant masses are much less important than the behavior in the range up to about $1 \mathrm{GeV}$ for the calculation of $a_{\mu}$. Thus, the very close results for $a_{\mu}$ coming from all the fits are not surprising. The range of the predicted values of $a_{\mu}$ within the class of models we examined is thus $79.4 \times 10^{11}<a_{\mu}^{P}<86.23 \times 10^{-11}$, if we take conservatively $3 \sigma$ errors, and the predicted value of $a_{\mu}$ is $(82.8 \pm 3.4) \times 10^{-11}$.

\section{IMPLEMENTATION OF THE MODEL IN EKHARA AND PHOKHARA GENERATORS}

The new transition pseudoscalar form factors were implemented in the event generator EKHARA [35,36]. As one can see from Fig. 1 the difference of the form factors from this paper as compared to the old model [9] for the configuration, where one of the invariant is equal to zero, is not big. Yet the experiments never have the second invariant mass equal to zero and the events are collected with a cut resulting from the cuts on the observed particles. The influence of this effect on the experimental side is a part of the systematic error. On the theory side it is model dependent with the part which is different from zero only when both photon virtualities are different from zero and thus never tested directly by any experiment in the spacelike region. The difference of the predictions of the influence of the second virtuality between the old and the new model is shown in Fig. 9. We plot there the relative difference of the differential cross sections calculated with the full form factors (full) and the case where one of the invariants was set to zero (approx.) as a function of the second invariant $Q^{2}=-(q-p)^{2} . q$ is the four-vector of the final positron and $p$ is the four-vector of the initial positron. We limit the invariant mass squared of the second virtual photon $\left[Q_{1}^{2}=-\left(q^{\prime}-p^{\prime}\right)^{2}\right.$, where $q^{\prime}$ is the fourvector of the final electron and $p^{\prime}$ is the four-vector of the initial electron] to $Q_{1}^{2} \leq 0.18 \mathrm{GeV}$ for $\pi^{0}$ and to $Q_{1}^{2} \leq 0.38$ for $\eta$ and $\eta^{\prime}$. As one can see, the corrections coming from the second invariant are by no means negligible, and their size exhibits the model dependence. In the plot the form factors of the fit 2 were used. For the fit 1 they look similar.

Having the model of the pseudoscalar transition form factors valid also in the timelike region, we are able to simulate the cross sections of the reactions $e^{+} e^{-} \rightarrow P \gamma$. This is done within the PHOKHARA Monte Carlo generator [33] framework. It is an upgrade of the version 9.2 [34] and will be available from the web page as release 9.3 [80]. Both options with the fit 1 and the fit 2 parameters are implemented. The next-to-leading order initial state radiative corrections were included based on the approach described in [81]. The virtual and the soft initial state corrections are universal and are exactly the same as in [81]; thus we do not repeat here the formulas. The matrix element describing the reaction $e^{+} e^{-} \rightarrow \pi^{0} \gamma \gamma$ was written as a product of the leptonic and hadronic current:

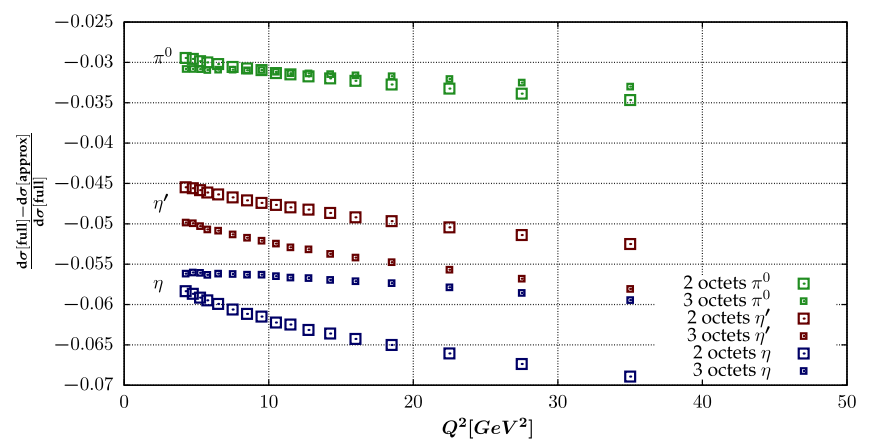

FIG. 9. The relative difference of differential cross sections calculated with $F_{\gamma^{*} \gamma^{*} P}\left(-Q^{2}, q_{1}^{2}\right)$ (full) and $F_{\gamma^{*} \gamma^{*} P}\left(-Q^{2}, 0\right)$ (approx.). See text for details. 

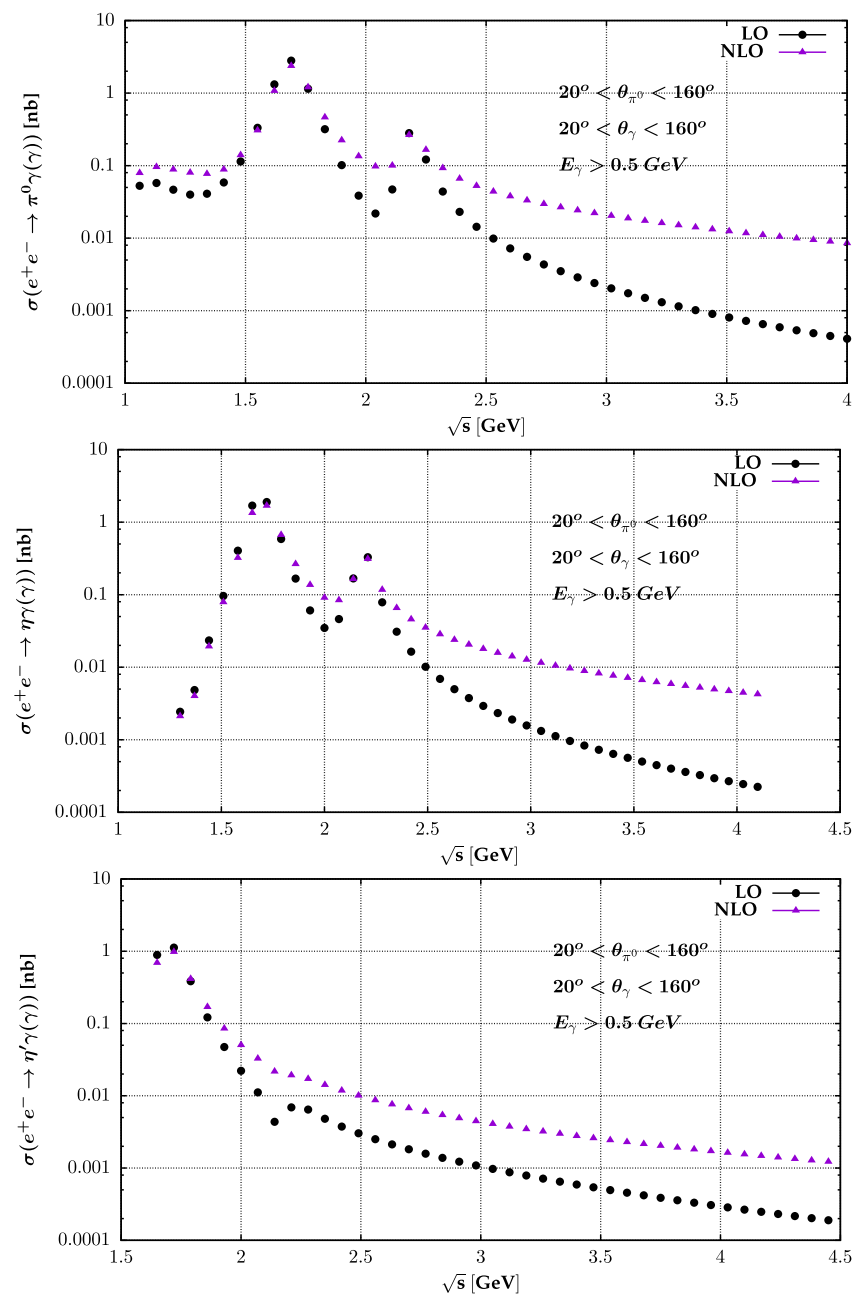

FIG. 10. Comparison between LO and NLO cross sections. See text for details.

$$
\begin{aligned}
\mathcal{M}\left[e^{+}\left(p_{1}\right) e^{-}\left(p_{2}\right)\right. & \left.\rightarrow \pi^{0}\left(q_{1}\right) \gamma\left(k_{1}\right) \gamma\left(k_{2}\right)\right] \\
& =L^{\nu}\left(k_{1}\right) H_{\nu}\left(k_{2}\right)+\left(k_{1} \leftrightarrow k_{2}\right)
\end{aligned}
$$

where

$$
H_{\nu}\left(k_{2}\right)=e^{2} \epsilon_{\mu \nu \alpha \beta} q_{1}^{\mu} k_{2}^{\alpha} \epsilon_{2}^{\beta} F_{\gamma^{*} \gamma^{*} P}\left(\left(q_{1}+k_{2}\right)^{2}, 0\right)
$$

and

$$
\begin{aligned}
L^{\nu}\left(k_{1}\right)= & \frac{i e^{2}}{2 p_{2} \cdot k_{1}} \bar{v}\left(p_{1}\right) \gamma^{\nu}\left(2 \epsilon_{1} p_{1}-\not k 1 \phi 1\right) u\left(p_{2}\right) \\
& +\frac{i e^{2}}{2 p_{1} \cdot k_{1}} \bar{v}\left(p_{1}\right)\left(\not 1 \not k 1-2 \epsilon_{1} p_{1}\right) \gamma^{\nu} u\left(p_{2}\right)
\end{aligned}
$$

with $\epsilon_{i}, i=1,2$ being a polarization vector of the photon with the four momentum $k_{i}$.

The effect of radiative corrections is shown in Fig. 10. The plots were obtained for fit 2 parameters accepting the events with the pseudoscalar particle and one of the photons with an energy bigger than $0.5 \mathrm{GeV}$ being observed within the angular range between 20 and 160 degrees. The radiative corrections are big due to the fact that the pseudoscalar transition form factor is falling fast at high values of the virtual photon mass. At leading order the form factor is calculated at $s$, while in the two photon amplitude it is calculated at much smaller invariants $Q^{2}=\left(q_{1}+k_{1}\right)^{2}$, or $Q^{2}=\left(q_{1}+k_{2}\right)^{2}$ resulting from the hard photon emission.

\section{CONCLUSIONS}

We model the Lagrangians $\mathcal{L}_{\gamma \gamma} P, \mathcal{L}_{\gamma V}, \mathcal{L}_{V \gamma P}$, and $\mathcal{L}_{V V P}$ within resonance chiral symmetric theory with $\mathrm{SU}(3)$ breaking. Two model versions with 22(17) couplings of the model are fitted to 536 experimental data points resulting in $\chi^{2}=415(454)$. Within the developed models we predict the pseudoscalar-exchange light-by-light contributions to the muon anomalous magnetic moment $a_{\mu}^{P}=(82.8 \pm 3.4) \times 10^{-11}$. The error covers also the model dependence within the class of models considered in this paper. The model was implemented into the Monte Carlo event generator EKHARA to simulate reactions $e^{+} e^{-} \rightarrow e^{+} e^{-} P,\left(P=\pi^{0}, \eta, \eta^{\prime}\right)$ and into the Monte Carlo event generator Phokhara to simulate reactions $e^{+} e^{-} \rightarrow$ $P \gamma(\gamma)$ at next-to-leading order.

\section{ACKNOWLEDGMENTS}

Work supported in part by the Polish National Science Centre, Grant No. DEC-2012/07/B/ST2/03867 and German Research Foundation Deutsche Forschungsgemeinschaft (DFG) under Contract No. Collaborative Research Center CRC-1044.
[1] S. Actis et al. (Working Group on Radiative Corrections and Monte Carlo Generators for Low Energies Collaboration), Eur. Phys. J. C 66, 585 (2010).

[2] G. W. Bennett et al. (Muon g-2 Collaboration), Phys. Rev. D 73, 072003 (2006).
[3] K. Hagiwara, A. D. Martin, D. Nomura, and T. Teubner, Phys. Lett. B 649, 173 (2007).

[4] M. Davier, A. Hoecker, B. Malaescu, and Z. Zhang, Eur. Phys. J. C 71, 1515 (2011); 72, 1874(E) (2012). 
[5] M. Benayoun, P. David, L. DelBuono, and F. Jegerlehner, Eur. Phys. J. C 75, 613 (2015).

[6] K. Hagiwara, A. Keshavarzi, A. D. Martin, D. Nomura, and T. Teubner, Nucl. Part. Phys. Proc. 287-288, 33 (2017).

[7] F. Jegerlehner, The Anomalous Magnetic Moment of the Muon (Springer, Cham, 2017), Vol. 274.

[8] M. Davier, A. Hoecker, B. Malaescu, and Z. Zhang, arXiv:1706.09436.

[9] H. Czyz, S. Ivashyn, A. Korchin, and O. Shekhovtsova, Phys. Rev. D 85, 094010 (2012).

[10] F. Jegerlehner and A. Nyffeler, Phys. Rep. 477, 1 (2009).

[11] M. Benayoun, P. David, L. DelBuono, and F. Jegerlehner, Eur. Phys. J. C 72, 1848 (2012).

[12] M. Benayoun, P. David, L. DelBuono, and F. Jegerlehner, Eur. Phys. J. C 73, 2453 (2013).

[13] T. Feldmann and P. Kroll, Eur. Phys. J. C 5, 327 (1998).

[14] P. Kroll and K. Passek-Kumericki, Phys. Rev. D 67, 054017 (2003).

[15] A. Scarpettini, D. G. Dumm, and N. N. Scoccola, Phys. Rev. D 69, 114018 (2004).

[16] S. S. Agaev and N. G. Stefanis, Phys. Rev. D 70, 054020 (2004).

[17] S. Noguera and S. Scopetta, Phys. Rev. D 85, 054004 (2012).

[18] P. Masjuan, Phys. Rev. D 86, 094021 (2012).

[19] N. G. Stefanis, A. P. Bakulev, S. V. Mikhailov, and A. V. Pimikov, Phys. Rev. D 87, 094025 (2013).

[20] S. Noguera and V. Vento, Eur. Phys. J. A 48, 143 (2012).

[21] X.-G. Wu, T. Huang, and T. Zhong, Chin. Phys. C 37, 063105 (2013).

[22] Y. Klopot, A. Oganesian, and O. Teryaev, Phys. Rev. D 87, 036013 (2013); 88, 059902(E) (2013).

[23] C.-Q. Geng and C.-C. Lih, Phys. Rev. C 86, 038201 (2012); 87, 039901(E) (2013).

[24] D. G. Dumm, S. Noguera, N. N. Scoccola, and S. Scopetta, Phys. Rev. D 89, 054031 (2014).

[25] J. P. B. C. de Melo, B. El-Bennich, and T. Frederico, FewBody Syst. 55, 373 (2014).

[26] H.-N. Li, Y.-L. Shen, and Y.-M. Wang, J. High Energy Phys. 01 (2014) 004.

[27] R. Escribano, P. Masjuan, and P. Sanchez-Puertas, Phys. Rev. D 89, 034014 (2014).

[28] S. S. Agaev, V. M. Braun, N. Offen, F. A. Porkert, and A. Schfer, Phys. Rev. D 90, 074019 (2014).

[29] R. Escribano, P. Masjuan, and P. Sanchez-Puertas, Eur. Phys. J. C 75, 414 (2015).

[30] R. Escribano, S. Gonzlez-Sols, P. Masjuan, and P. SanchezPuertas, Phys. Rev. D 94, 054033 (2016).

[31] T. Zhong, X.-G. Wu, and T. Huang, Eur. Phys. J. C 76, 390 (2016).

[32] D. G. Dumm, S. Noguera, and N. N. Scoccola, Phys. Rev. D 95, 054006 (2017).

[33] G. Rodrigo, H. Czyz, J. H. Kühn, and M. Szopa, Eur. Phys. J. C 24, 71 (2002).

[34] H. Czyz, J. H. Kühn, and S. Tracz, Phys. Rev. D 94, 034033 (2016).

[35] H. Czyz and S. Ivashyn, Comput. Phys. Commun. 182, 1338 (2011).

[36] H. Czyz and E. Nowak-Kubat, Phys. Lett. B 634, 493 (2006).
[37] T. Feldmann, Int. J. Mod. Phys. A 15, 159 (2000).

[38] T. Feldmann, P. Kroll, and B. Stech, Phys. Rev. D 58, 114006 (1998).

[39] J. Wess and B. Zumino, Phys. Lett. 37B, 95 (1971).

[40] E. Witten, Nucl. Phys. B223, 422 (1983).

[41] J. Prades, Z. Phys. C 63, 491 (1994); 11, 571(E) (1999).

[42] S. Uehara et al. (Belle Collaboration), Phys. Rev. D 86, 092007 (2012).

[43] H. J. Behrend et al. (CELLO Collaboration), Z. Phys. C 49, 401 (1991).

[44] J. Gronberg et al. (CLEO Collaboration), Phys. Rev. D 57, 33 (1998).

[45] M. N. Achasov et al., Phys. Rev. D 74, 014016 (2006).

[46] M. N. Achasov et al. (SND Collaboration), Phys. Rev. D 93, 092001 (2016).

[47] R. R. Akhmetshin et al. (CMD-2 Collaboration), Phys. Lett. B 605, 26 (2005).

[48] P. Adlarson et al. (A2 Collaboration), Phys. Rev. C 95, 025202 (2017).

[49] P. Adlarson et al., Phys. Rev. C 95, 035208 (2017).

[50] M. Ablikim et al. (BESIII Collaboration), Phys. Rev. D 92, 012001 (2015).

[51] A. Anastasi et al. (KLOE-2 Collaboration), Phys. Lett. B 757, 362 (2016).

[52] D. Babusci et al. (KLOE-2 Collaboration), Phys. Lett. B 742, 1 (2015).

[53] B. M. K. Nefkens et al. (A2 at MAMI Collaboration), Phys. Rev. C 90, 025206 (2014).

[54] C. Patrignani et al. (Particle Data Group Collaboration), Chin. Phys. C 40, 100001 (2016).

[55] B. Aubert et al. (BABAR Collaboration), Phys. Rev. D 80, 052002 (2009).

[56] R. Arnaldi et al. (NA60 Collaboration), Phys. Lett. B 757, 437 (2016).

[57] P. del Amo Sanchez et al. (BABAR Collaboration), Phys. Rev. D 84, 052001 (2011).

[58] B. Aubert et al. (BABAR Collaboration), Phys. Rev. D 74, 012002 (2006).

[59] G. P. Lepage and S. J. Brodsky, Phys. Rev. D 22, 2157 (1980).

[60] G. J. Gounaris and J. J. Sakurai, Phys. Rev. Lett. 21, 244 (1968).

[61] J. Bijnens, E. Gamiz, E. Lipartia, and J. Prades, J. High Energy Phys. 04 (2003) 055.

[62] V. A. Novikov, M. A. Shifman, A. I. Vainshtein, M. B. Voloshin, and V. I. Zakharov, Nucl. Phys. B237, 525 (1984).

[63] L. Ametller, J. Bijnens, A. Bramon, and F. Cornet, Phys. Rev. D 45, 986 (1992).

[64] C. Hanhart, A. Kupść, U. G. Meiner, F. Stollenwerk, and A. Wirzba, Eur. Phys. J. C 73, 2668 (2013); 75, 242(E) (2015).

[65] R. M. Drees et al. (SINDRUM-I Collaboration), Phys. Rev. D 45, 1439 (1992).

[66] F. Farzanpay et al., Phys. Lett. B 278, 413 (1992).

[67] H. Berghauser et al., Phys. Lett. B 701, 562 (2011).

[68] G. Usai (NA60 Collaboration), Nucl. Phys. A855, 189 (2011).

[69] C. Lazzeroni et al. (NA62 Collaboration), Phys. Lett. B 768, 38 (2017).

[70] K. Melnikov and A. Vainshtein, Phys. Rev. D 70, 113006 (2004).

[71] K. Kampf and J. Novotny, Phys. Rev. D 84, 014036 (2011). 
[72] P. Roig, A. Guevara, and G. L. Castro, Phys. Rev. D 89, 073016 (2014).

[73] A. Nyffeler, Phys. Rev. D 94, 053006 (2016).

[74] M. Hayakawa and T. Kinoshita, Phys. Rev. D 57, 465 (1998); 66, 019902(E) (2002).

[75] M. Knecht and A. Nyffeler, Phys. Rev. D 65, 073034 (2002).

[76] J. Bijnens, E. Pallante, and J. Prades, Nucl. Phys. B626, 410 (2002).

[77] A. E. Dorokhov and W. Broniowski, Phys. Rev. D 78, 073011 (2008).
[78] A. Nyffeler, Phys. Rev. D 79, 073012 (2009).

[79] A. Grardin, H. B. Meyer, and A. Nyffeler, Phys. Rev. D 94, 074507 (2016).

[80] http://ific.uv.es/ rodrigo/phokhara/.

[81] H. Czyz, M. Gunia, and J. H. Kühn, J. High Energy Phys. 08 (2013) 110.

Correction: The license statement "Funded by SCOAP" was missing on the first page of the article and has been inserted. 